TRANSACTIONS OF THE

AMERICAN MATHEMATICAL SOCIETY

Volume 351 , Number 12 , Pages 4791-4822

S 0002-9947(99)02189-3

Article electronically published on August 23, 1999

\title{
MÖBIUS-LIKE GROUPS OF HOMEOMORPHISMS OF THE CIRCLE
}

\author{
NATAŠA KOVAČEVIĆ
}

\begin{abstract}
An orientation preserving homeomorphism of $S^{1}$ is Möbius-like if it is conjugate in $\mathrm{Homeo}\left(S^{1} 1\right)$ to a Möbius transformation. Our main result is: given a (noncyclic) group $G \hookrightarrow H o m e o_{+}\left(S^{1}\right)$ whose every element is Möbiuslike, if $G$ has at least one global fixed point, then the whole group $G$ is conjugate in Homeo $\left(S^{1} 1\right)$ to a Möbius group if and only if the limit set of $G$ is all of $S^{1}$. Moreover, we prove that if the limit set of $G$ is not all of $S^{1}$, then after identifying some closed subintervals of $S^{1}$ to points, the induced action of $G$ is conjugate to an action of a Möbius group. Said differently, $G$ is obtained from a group which is conjugate to a Möbius group, by a sort of generalized Denjoy's insertion of intervals. In this case $G$ is isomorphic, as a group, to a Möbius group.

This result has another interpretation. Namely, we prove that a group $G$ of orientation preserving homeomorphisms of $\boldsymbol{R}$ whose every element can be conjugated to an affine map (i.e., a map of the form $x \mapsto a x+b$ ) is just the conjugate of a group of affine maps, up to a certain insertion of intervals. In any case, the group structure of $G$ is the one of an affine group.
\end{abstract}

\section{INTRODUCTION}

Denote by $\mathcal{M}$ the group of orientation preserving Möbius transformations of the complex plane preserving the unit disc. Every element of $\mathcal{M}$ also preserves the boundary, $S^{1}$, of the unit disc. In other words, we can view $\mathcal{M}$ as a subgroup of the group of all orientation preserving homeomorphisms of $S^{1}$ via

$$
\begin{gathered}
\mathcal{M} \hookrightarrow \text { Homeo }_{+}\left(S^{1}\right) \\
\left.f \longmapsto f\right|_{S^{1}},
\end{gathered}
$$

where $\left.f\right|_{S^{1}}$ denotes the restriction of $f$ to $S^{1}$. Throughout this paper we will refer to Möbius groups as subgroups of $\mathcal{M}$, and we will mostly think of them in terms of their restrictions to $S^{1}$.

Definition. Given $f \in \mathrm{Homeo}_{+}\left(S^{1}\right)$ we say that $f$ is Möbius-like if there exists some homeomorphism $g$ of $S^{1}$ such that $g f g^{-1} \in \mathcal{M}$.

Equivalently, $f$ is Möbius-like if it has the dynamics of a Möbius transformation. ${ }^{1}$

In this paper we investigate groups of Möbius-like homeomorphisms of the circle. Our main result is the following theorem.

Main Theorem. Let $G$ be a group of orientation preserving homeomorphisms of $S^{1}$ such that every element of $G$ is Möbius-like. Let $L(G)$ denote the limit set of

Received by the editors March 7, 1995 and, in revised form, July 31, 1997.

1991 Mathematics Subject Classification. Primary 57S05.

${ }^{1}$ This equivalence will be discussed in Section 2 . 
G. ${ }^{2}$ Suppose there exists a point on $S^{1}$ which is fixed by all elements of $G$. Then exactly one of the following possibilities occurs:

1. $G$ is cyclic.

2. $L(G)=S^{1}$. Then the whole group $G$ can be conjugated to a nondiscrete Möbius group (i.e., its restriction to $S^{1}$ ).

3. $L(G) \neq S^{1}$. Then $S^{1}-L(G)$ is an infinite union of disjoint open intervals $\left(x_{i}, y_{i}\right)$. There is a new circle $S_{\star}^{1}$, which is obtained from $S^{1}$ by identifying intervals $\left[x_{i}, y_{i}\right]$ to points, and there is an induced action of $G$ on $S_{\star}^{1}$ so that $G$ with this induced action is as in 2. In particular, $G$ is isomorphic (as a group) to a Möbius group.

Simply speaking, this theorem says that a group with a global fixed point and whose every element has the dynamics of a Möbius map has to be the conjugate of a Möbius group up to a sort of generalized Denjoy's insertion of intervals.

Remark. There is an alternative way of saying all of the above by using the standard map which maps the unit disc onto the upper half-plane. Then one can talk about groups of orientation preserving homeomorphisms of $\boldsymbol{R} \cup \infty$. This is particularly appropriate in the situation described in the Main Theorem, where there is a point which is fixed by all elements of the group. We can assume that point is $\infty$, and thus we are dealing with a group of orientation preserving homeomorphisms of $\boldsymbol{R}$. The notion of Möbius-likeness on the circle then translates to the notion of affine-likeness on $\boldsymbol{R}$ (for details see the last paragraph of section 1).

Main Theorem (alternative version). Suppose $G$ is a group of orientation preserving homeomorphisms of $\boldsymbol{R}$, such that every element of $G$ can be conjugated (in Homeo $(\boldsymbol{R})$ ) to an affine map. Then exactly one of the following possibilities occurs:

1. $G$ is cyclic.

2. There exists $x \in \boldsymbol{R}$ with dense $G$-orbit. Then the whole group $G$ can be conjugated to a nondiscrete affine group.

3. There exists $x \in G$ whose $G$-orbit is not dense in $\boldsymbol{R}$. The complement of the closure of the $G$-orbit of $x$ is an infinite union of disjoint open intervals $\left(x_{i}, y_{i}\right)$. There is a new copy of $\boldsymbol{R}$, call it $\boldsymbol{R}_{\star}$, which is obtained from $\boldsymbol{R}$ by identifying intervals $\left[x_{i}, y_{i}\right]$ to points, and there is an induced action of $G$ on $\boldsymbol{R}_{\star}$ so that $G$ with this induced action is as in 2. In particular, $G$ is isomorphic (as a group) to an affine group.

The proof of the main theorem relies on the following result with a long history.

Theorem (Tukia, Hinkkanen, Gabai, Casson-Jungreis). Group $G \hookrightarrow \mathrm{Homeo}_{+}\left(S^{1}\right)$ is conjugate in $\operatorname{Homeo}\left(S^{1}\right)$ to a Möbius group if and only if $G$ is a convergence group.

By putting together the results given in say $[\mathrm{G}],[\mathrm{T}]$ and $[\mathrm{H}]$ one obtains the complete proof of this theorem.

The proof of the main theorem is exposed in the following way. In section 1 we review some basic definitions and known facts, and we prove some technical lemmas. The main theorem deals with groups of Möbius-like homeomorphisms which all share a fixed point, i.e., groups with at least one global fixed point on

\footnotetext{
${ }^{2}$ The standard notion of the limit set makes sense for groups of Möbius-like maps, see Definition 1.6 .
} 
$S^{1}$. (It should be noted here that a group of Möbius-like homeomorphisms of the circle can have 0,1 or 2 global fixed points.) In section 2 we give a proof of the main theorem in the case of groups with two global fixed points (Theorems 2.1 and 2.4). In section 3 we do the same for groups with exactly one global fixed point (Theorems 3.1 and 3.3). In both cases, the above theorem helps us in the sense that we only need to find out when the groups in question are convergence groups.

\section{Preliminary Definitions and observations}

Throughout this paper we restrict ourselves to orientation preserving homeomorphisms of the circle only.

View $S^{1}$ as being oriented counterclockwise; therefore $(x, y)$ denotes all the points counterclockwise from $x$ to $y$. We define $[x, y),(x, y],[x, y]$ similarly. Given two open intervals $(x, y)$ and $(u, v)$ on $S^{1}$ we write $(x, y) \propto(u, v)$ if either $x<u<y<$ $v<x$ or $u<x<v<y<x$ (see Figure 1.1).

The universal cover of $S^{1}$ is $\boldsymbol{R}$ via covering map $x \mapsto e^{2 \pi i x}, x \in \boldsymbol{R}$. By abuse of notation, we denote all lifts to $\boldsymbol{R}$ of a point $x \in S^{1}$ by the same symbol $x$.

Given $f \in \mathrm{Homeo}_{+}\left(S^{1}\right)$, it can be lifted to a homeomorphism $\tilde{f}: \boldsymbol{R} \rightarrow \boldsymbol{R}$ so that $f\left(e^{2 \pi i x}\right)=e^{2 \pi i \tilde{f}(x)}, \forall x \in \boldsymbol{R}$. Since $f$ is orientation preserving, $\tilde{f}$ is a strictly increasing function. There are many different lifts of $f$, but we will most commonly take $\tilde{f}$ to be the lift of $f$ whose graph is "the closest" to the line $y=x$. Actually, by abuse of notation we will use $f$ for $\tilde{f}$.

Many arguments in this paper deal with functions $S^{1} \rightarrow S^{1}$ which are limits of sequences of homeomorphisms of $S^{1}$. It is therefore natural to introduce the following convention about drawing the graphs of such functions (i.e., their lifts to $\boldsymbol{R})$ : if say a function $f$ is a limit function of some sequence $\left\{f_{n}\right\}$ in $\mathrm{Homeo}_{+}\left(S^{1}\right)$, and $f$ has a jump discontinuity at some point $x$, then we will draw a vertical segment between points $\left(x, f_{-}(x)\right)$ and $\left(x, f_{+}(x)\right)$ as a part of the graph of $f$.

Definition 1.1. A group $G \hookrightarrow \mathrm{Homeo}_{+}\left(S^{1}\right)$ is discrete if it is discrete in the compact-open topology of $\mathrm{Homeo}_{+}\left(S^{1}\right)$. Equivalently, $G$ is discrete if no sequence of distinct elements in $G$ converges (uniformly) to the identity map.

The group of all orientation preserving Möbius transformations of the complex plane $\boldsymbol{C}$, which preserve the unit disc $\bar{D}=\{z \in \boldsymbol{C}|| z \mid \leq 1\}$, is denoted by $\mathcal{M}$.
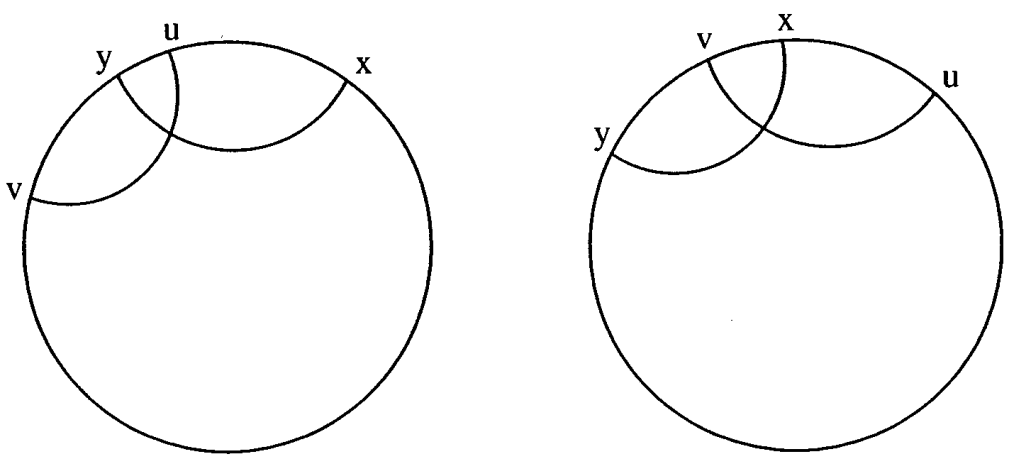

Figure 1.1 
Every element of $\mathcal{M}$ is of the form

$$
z \mapsto \frac{a z+\bar{c}}{c z+\bar{a}}
$$

where $a$ and $c$ are complex numbers such that $|a|^{2}-|c|^{2}=1$. The group $\mathcal{M}$ is exactly the group of orientation preserving isometries of $\boldsymbol{H}^{2}$ in the open unit disc model.

There are three types of elements of $\mathcal{M}$ which we distinguish by looking at their fixed points on $S^{1}$ : hyperbolic (transformations having two fixed points on $S^{1}$, one attractive, one repulsive; these two points correspond to the endpoints on $S^{1}$ of the hyperbolic axis of the transformation.); parabolic (transformations having one fixed point on $S^{1}$ ); elliptic (transformations having no fixed points on $S^{1}$ ).

Definition 1.2. Given $f \in \mathrm{Homeo}_{+}\left(S^{1}\right)$ we say that $f$ is Möbius-like if there exists some homeomorphism $g$ of $S^{1}$ such that $g f g^{-1} \in \mathcal{M}$.

Topological characterization of Möbius-like maps. ${ }^{3}$ The number of fixed points is invariant under conjugation, so Möbius-like homeomorphisms can have two, one or no fixed points on $S^{1}$. Accordingly we have the same characterization as for Möbius transformations: a Möbius-like homeomorphism of $S^{1}$ can be either hyperbolic, or parabolic or elliptic. However, there is more to the topology of Möbius-like maps than just the number of fixed points, as the following characterization shows. Let $f \in \mathrm{Homeo}_{+}\left(S^{1}\right), f \neq i d$.

$f$ has two fixed points on $S^{1}$ : If one fixed point is attractive, usually denoted $P_{f}$, and the other is repulsive, usually denoted $N_{f}$, then $f$ is Möbius-like hyperbolic. In this case we refer to the pair $\left\{N_{f}, P_{f}\right\}$ as the axis of $f$.

$f$ has one fixed point on $S^{1}$ : Then $f$ is necessarily Möbius-like parabolic.

$f$ has no fixed points on $S^{1}$ : Then things are more complicated. If $f$ has finite order, i.e., $f^{n}=i d$ for some $n$, then $f$ is Möbius-like elliptic. Now, if $f$ has infinite order, then $f$ needs to satisfy some additional condition in order to be Möbius-like, as demonstrated by Denjoy's construction which we recall below. For example, if the $f$-orbit of some point $x \in S^{1}$ is dense in $S^{1}$, then $f$ is Möbius-like elliptic of infinite order.

Denjoy's construction. ${ }^{4}$ Start with a genuine rotation $f$ of infinite order. Say $f(z)=z e^{2 \pi i \theta}$ with $\theta$ irrational. Choose any $x \in S^{1}$. Then the $f$-orbit of $x, o(x)$, is a countable dense set in $S^{1}$. Now construct a new, bigger circle $\overline{S^{1}}$ by inserting a closed interval at each point of $o(x)$, taking care that the total sum of the lengths of the inserted intervals is finite. See Figure 1.2.

$f$ induces a homeomorphism $\bar{f}: \overline{S^{1}} \rightarrow \overline{S^{1}}$ in the following way: if a point $z \in \overline{S^{1}}$ was untouched by the construction, i.e., no interval was inserted at $z$, set $\bar{f}(z)=f(z)$; to define $\bar{f}$ on the interval inserted at a point $z \in o(x)$, choose any orientation preserving homeomorphism mapping that interval to the interval inserted at the point $f(z) \in o(x)$. Now, $\bar{f}$ has no periodic points, so it cannot be conjugated to a finite order Möbius transformation. On the other hand, it cannot be conjugated to an irrational rotation of $\overline{S^{1}}$ either because there are $\bar{f}$-orbits which are not dense in $\overline{S^{1}}$, and that is a property which is invariant under conjugation.

\footnotetext{
${ }^{3}$ A proof of this characterization can be found in e.g. [T].

${ }^{4}$ See [D] for more subtleties of the construction, e.g., concerning differentiability.
} 

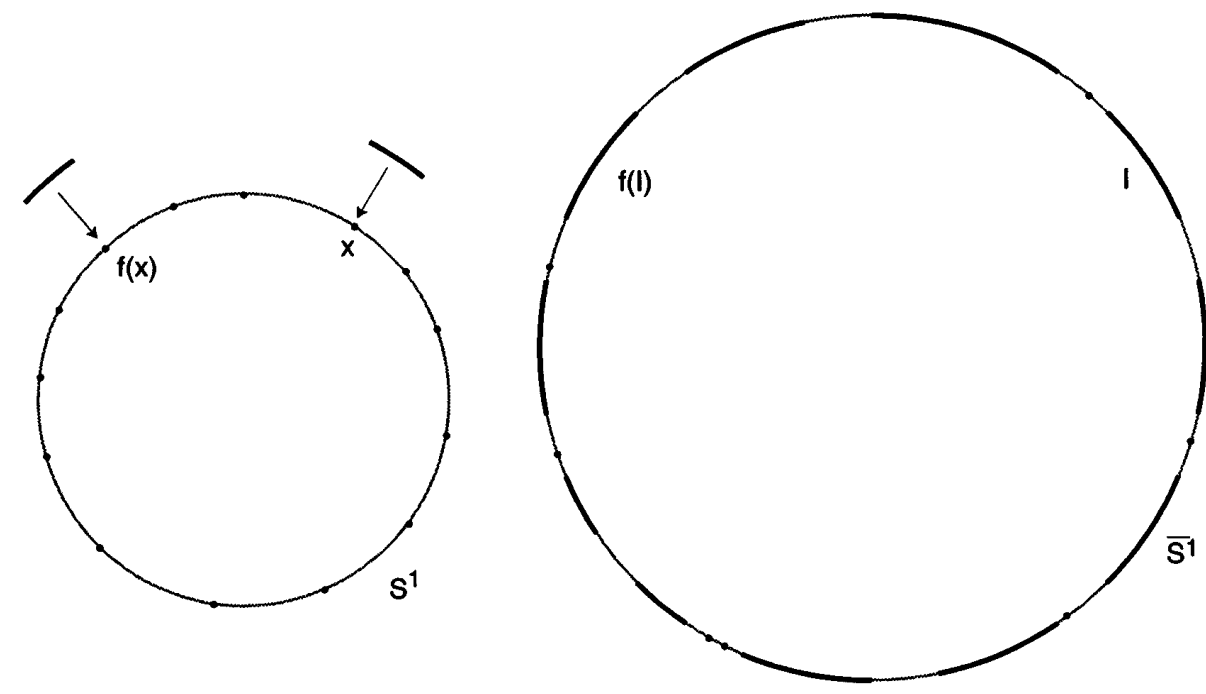

FiguRE 1.2

As mentioned in the Introduction, there is a close connection with convergence groups.

Definition 1.3 (Gehring-Martin). ${ }^{5}$ Let $G$ be a subgroup of $\mathrm{Homeo}_{+}\left(S^{1}\right)$. G is a convergence group if every sequence $\left\{f_{n}\right\}$ in $G$ has a subsequence $\left\{f_{n_{i}}\right\}$ such that either:

a) $\exists x, y \in S^{1}$ such that

$f_{n_{i}} \rightarrow y$ uniformly on compact subsets of $S^{1}-\{x\}$,

$f_{n_{i}}^{-1} \rightarrow x$ uniformly on compact subsets of $S^{1}-\{y\}$, or

b) $\exists, f \in$ Homeo+ $_{+}\left(S^{1}\right)$ such that

$f_{n_{i}} \rightarrow f$ uniformly on $S^{1}$,

$f_{n_{i}}^{-1} \rightarrow f^{-1}$ uniformly on $S^{1}$.

In terms of lifts of $f_{n_{i}}$ 's, condition a) means that the graphs of the $f_{n_{i}}$ 's approach a sort of step function consisting of jumps and flats ${ }^{6}$ of length 1. See Figure 1.3.

Observation 1.4. In the above definition one can replace conditions a) and b) by:

a') $\exists x, y \in S^{1}$ such that

$f_{n_{i}} \rightarrow y$ pointwise on $S^{1}-\{x\}$,

$f_{n_{i}}^{-1} \rightarrow x$ pointwise on $S^{1}-\{y\}$.

b') $\exists f \in \mathrm{Homeo}_{+}\left(S^{1}\right)$ such that

$f_{n_{i}} \rightarrow f$ pointwise on $S^{1}$,

$f_{n_{i}}^{-1} \rightarrow f^{-1}$ pointwise on $S^{1}$.

Lemma 1.5. Let $\left\{f_{n}\right\}$ be a sequence in $\mathrm{Homeo}_{+}\left(S^{1}\right)$ such that $f_{n} \rightarrow f$ pointwise on $S^{1}$, where $f \in$ Homeo $_{+}\left(S^{1}\right)$. Then $f_{n} \rightarrow f$ uniformly on $S^{1}$.

\footnotetext{
${ }^{5}$ In the original definition given in [G-M] $G$ is assumed to be a subgroup of $\operatorname{Homeo}\left(S^{1}\right)$.

${ }^{6} \mathrm{~A}$ flat of a function is a part of its graph where the function is constant.
} 


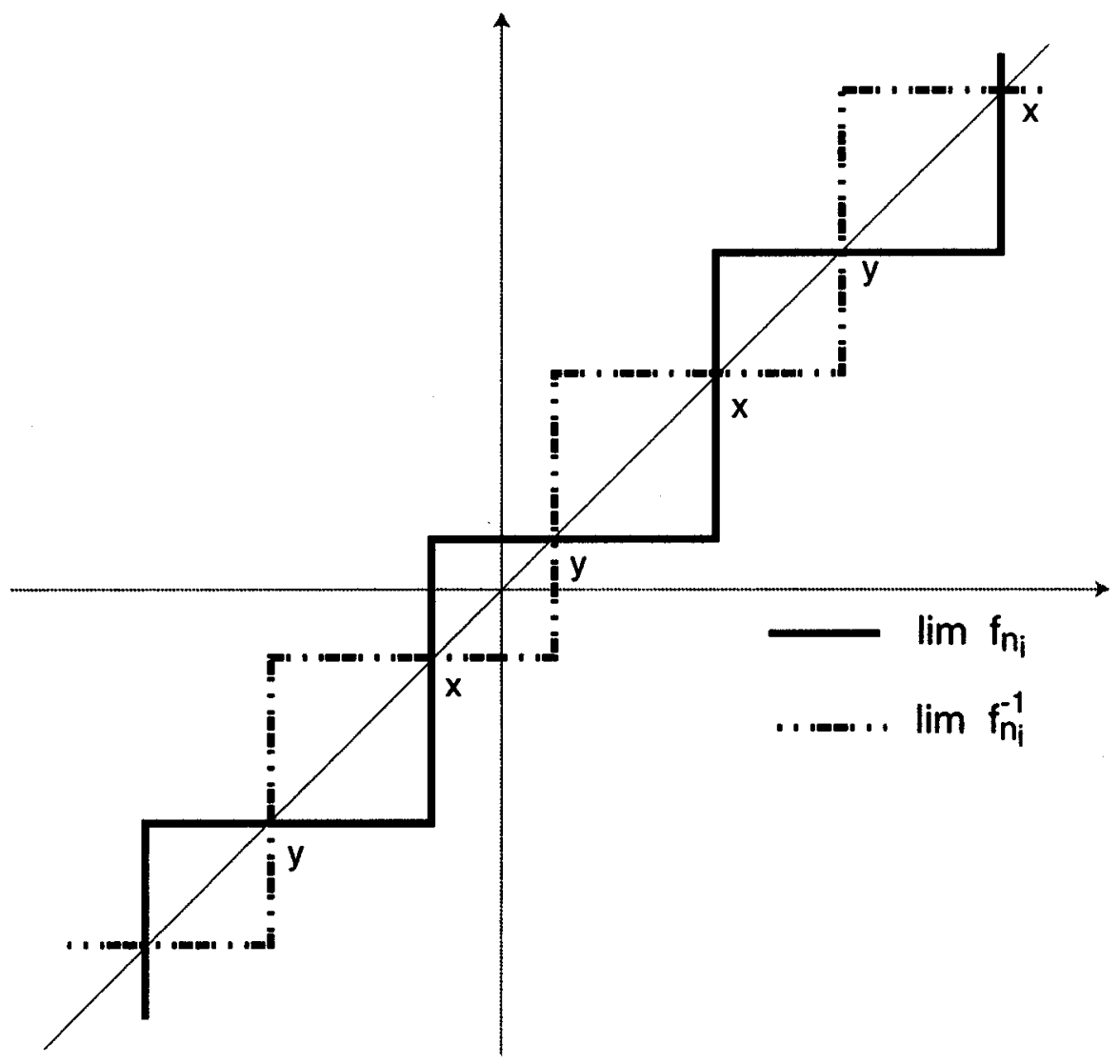

Figure 1.3

Proof of Lemma 1.5. Consider the lifts of the $f_{n}$ 's and $f$ on $[0,1]$. Given $\varepsilon>0$, uniform continuity of $f$ gives us $0<\delta<\varepsilon / 2$ such that

$$
\forall x, y \in \boldsymbol{R}, \quad \text { if }|x-y|<\delta, \text { then }|f(x)-f(y)|<\varepsilon / 2 .
$$

Partition interval $[0,1]$ by points $0=x_{0}, x_{1}, \ldots, x_{n-1}, x_{n}=1$, so that $\left|x_{i+1}-x_{i}\right|<\delta$ for all $i$. Then find $n_{0}$ such that $\forall n, \geq n_{0}, \forall i,\left|f_{n}\left(x_{i}\right)-f\left(x_{i}\right)\right|<\delta$. Consequently, for every $z \in\left(x_{i}, x_{i+1}\right)$ we have

$f\left(x_{i}\right)-\delta<f_{n}\left(x_{i}\right)<f_{n}(z)<f_{n}\left(x_{i+1}\right)<f\left(x_{i+1}\right)+\delta$ and $f\left(x_{i}\right)<f(z)<f\left(x_{i+1}\right)$, because lifts of $f_{n}$ 's and $f$ are increasing functions on $\boldsymbol{R}$. Thus

$\left|f(z)-f_{n}(z)\right|<f\left(x_{i+1}\right)+\delta-f\left(x_{i}\right)+\delta=f\left(x_{i+1}\right)-f\left(x_{i}\right)+2 \delta<\varepsilon / 2+\varepsilon / 2=\varepsilon$.

Proof of Observation 1.4. $\left.\mathrm{b}^{\prime}\right) \Rightarrow \mathrm{b}$ ) is clear by Lemma 1.5.

$\left.\mathrm{a}^{\prime}\right) \Rightarrow$ a). In order to simplify the notation, assume $f_{n} \rightarrow y$ pointwise on $S^{1}-\{x\}$ and $f_{n}^{-1} \rightarrow x$ pointwise on $S^{1}-\{y\}$. We want to show that the first convergence is uniform on compact subsets of $S^{1}-\{x\}$, so choose a closed interval $[a, b] \subset S^{1}-\{x\}$. Given $\varepsilon>0$ find $n_{0}$ such that $f_{n}(a), f_{n}(b) \in(y-\varepsilon, y+\varepsilon)$, for all $n \geq n_{0}$. It suffices to show that $f_{n}[a, b] \subset(y-\varepsilon, y+\varepsilon)$ for all but finitely many $n \geq n_{0}$.

Suppose the contrary, that there exists a subsequence $\left\{f_{n_{i}}\right\}$ (with $n_{i} \geq n_{0}$ for all $i)$ such that $f_{n_{i}}[a, b] \not \subset(y-\varepsilon, y+\varepsilon)$ (equivalently $f_{n_{i}}[b, a] \subset(y-\varepsilon, y+\varepsilon)$ ) for all $i$. 
By passing to a subsequence we can assume that sequences $\left\{f_{n_{i}}(a)\right\}$ and $\left\{f_{n_{i}}(b)\right\}$ are monotonic and such that either:

(i) $f_{n_{i}}[b, a] \supset f_{n_{i+1}}[b, a]$ for all $i$, or

(ii) $f_{n_{i}}[b, a] \cap f_{n_{i+1}}[b, a]=\emptyset$ for all $i$.

In both cases $\exists c \in\{a, b\}$ such that $f_{n_{i}}(c) \neq y$ for all $i$. Set $z=f_{n_{1}}(c)$.

In case (i) we have $f_{n_{i}}^{-1} f_{n_{1}}[b, a] \supset[b, a]$ for all $i$. Equivalently, $f_{n_{i}}^{-1} f_{n_{1}}[a, b] \subset[a, b]$. In particular, $f_{n_{i}}^{-1}(z) \in[a, b]$ for all $i$. But this contradicts $f_{n}^{-1} \rightarrow x$ pointwise on $S^{1}-\{y\}$, since $[a, b] \cap\{x\}=\emptyset$.

In case (ii) $f_{n_{i}}[b, a] \cap f_{n_{1}}[b, a]=\emptyset$ for all $i$, or equivalently $f_{n_{i}}^{-1} f_{n_{1}}[b, a] \subset(a, b)$. So again we get $f_{n_{i}}^{-1}(z) \in(a, b)$ for all $i$. Contradiction.

Definition 1.6. Given a group $G \hookrightarrow \mathrm{Homeo}_{+}\left(S^{1}\right)$, the limit set of $G$, denoted $L(G)$, is the set of all $x \in S^{1}$ such that $G$ does not act properly discontinuously at $x$ (i.e., for every neighborhood $U$ of $x$ there exist infinitely many elements $g \in G$ such that $g(U) \cap U \neq \emptyset)$. It is easy to see that $L(G)$ is a closed $G$-invariant subset of $S^{1}$.

It is sometimes convenient to view $S^{1}$ as $\boldsymbol{R} \cup\{\infty\}$ and $D$ as the upper halfplane $H=\{z \in \boldsymbol{C} \mid \operatorname{Im}(z)>0\}$. Then $\mathcal{M}$ becomes $P S L_{2}(\boldsymbol{R})$, i.e., the group of transformations of the form

$$
z \mapsto \frac{a z+b}{c z+d}, \quad z \in H,
$$

where $a, b, c, d \in \boldsymbol{R}$, and $a d-b c=1$. Accordingly, we have a faithful inclusion

$$
\begin{aligned}
P S L_{2}(\boldsymbol{R}) & \hookrightarrow \mathrm{Homeo}_{+}(\boldsymbol{R} \cup\{\infty\}), \\
f & \left.\longmapsto f\right|_{\boldsymbol{R} \cup\{\infty\} .}
\end{aligned}
$$

The subgroup of all elements of $P S L_{2}(\boldsymbol{R})$ fixing $\infty$ is $\mathcal{A}$, the group of all affine homeomorphisms of $\boldsymbol{R}$ (i.e., maps of the form $x \mapsto a x+b$ ). Accordingly, an affine group is any subgroup of $\mathcal{A}$.

Definition 1.7. By analogy we say that $f \in \mathrm{Homeo}_{+}(\boldsymbol{R})$ is affine-like if there exists a homeomorphism $g$ of $\boldsymbol{R}$ such that $g f g^{-1} \in \mathcal{A}$.

\section{Groups With TWO GLOBAL FIXED POINTS}

Theorem 2.1. Let $G \hookrightarrow H_{\text {omeo+ }}\left(S^{1}\right)$ be a group whose every element is Möbiuslike. Suppose $G$ has two global fixed points a and $b$; in other words, $G$ is a purely hyperbolic group whose every nontrivial element is a hyperbolic Möbius-like map with $\{a, b\}$ as its axis. Assume also that $G$ is not cyclic. ${ }^{7}$ Then $G$ is a convergence group if and only if $L(G)=S^{1}$.

Lemma 2.2. Suppose $G$ is as above. If $L(G)=S^{1}$, then for every $x \in(a, b)$ (respectively $(b, a))$ the orbit of $x$ under $G$ is dense in $[a, b]$ (respectively $[b, a])$.

Proof of Lemma 2.2. Let $x \in(a, b)$. Denote by $o(x)$ the $G$-orbit of $x$. Suppose $o(x)$ is not dense in $[a, b]$, i.e., $[a, b]-\overline{o(x)} \neq \emptyset$. Note that, for every nontrivial $h \in G$, with say $N_{h}=a$ and $P_{h}=b$,

$$
h^{n}(x) \rightarrow b \text { and } h^{-n}(x) \rightarrow a, \text { as } n \rightarrow \infty .
$$

${ }^{7}$ If $G$ is cyclic, then we know that $G$ is a convergence group; see the topological characterization of the Möbius-like maps given in section 1 . 


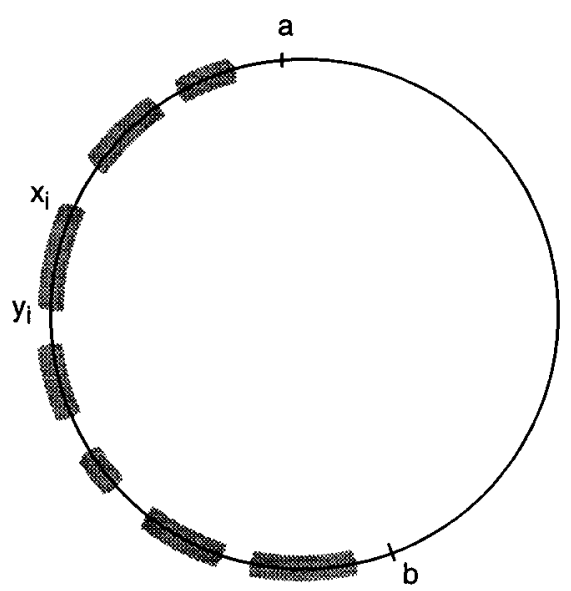

FiguRE 2.1

Hence $a, b \in \overline{o(x)}$. Consequently, $[a, b]-\overline{o(x)}$ is an infinite union of disjoint open intervals $\left(x_{i}, y_{i}\right), i=1,2, \ldots$, where $a<x_{i}<y_{i}<b$ and $x_{i}, y_{i} \in \overline{o(x)}$ for all $i$. See Figure 2.1.

Fix some $i . L(G)=S^{1}$ implies the existence of some $g \in G, g \neq i d$, such that $g\left(x_{i}, y_{i}\right) \cap\left(x_{i}, y_{i}\right) \neq \emptyset$.

We can have neither $g\left(x_{i}, y_{i}\right) \subset\left(x_{i}, y_{i}\right)$ nor $g\left(x_{i}, y_{i}\right) \supset\left(x_{i}, y_{i}\right)$ since that would mean that $g$ has at least one fixed point in $\left[x_{i}, y_{i}\right]$. Hence $g\left(x_{i}, y_{i}\right) \curvearrowright\left(x_{i}, y_{i}\right)$. But this contradicts the definition of $x_{i}, y_{i}$.

Proof of Theorem 2.1. $(\Rightarrow)$ Suppose $G$ is a convergence group. Then $G$ is a Möbius group up to conjugation. We can actually assume that $G$ is a genuine Möbius group since the condition $L(G)=S^{1}$ is preserved under conjugation. It is an easy fact that a purely hyperbolic Möbius group with two global fixed points is discrete if and only if it is not cyclic, i.e., it cannot be generated by a single transformation. Since we have assumed that $G$ is not cyclic, it follows that $G$ is non-discrete, and hence $L(G)=S^{1}$

$(\Leftarrow)$ Suppose $L(G)=S^{1}$, and let us prove that $G$ is a convergence group. Note that given any two distinct elements $g, h \in G$, the graphs of $g$ and $h$ intersect at points $a$ and $b$ and are disjoint on $(a, b) \cup(b, a)$. Indeed, if they had an intersection at some point $x \neq a, b$, that would mean that $g(x)=h(x)$. Hence the transformation $g^{-1} h$ would have $x$ as its third fixed point (besides $a$ and $b$ ), which is a contradiction.

Let $\left\{f_{n}\right\}$ be a sequence of distinct elements of $G$. The graphs of the $f_{n}$ 's are pairwise disjoint on $(a, b) \cup(b, a)$. Therefore, after passing to a subsequence, we can assume that $\left\{f_{n}\right\}$ is strictly monotonic on both $(a, b)$ and $(b, a)$, only in the opposite sense. More precisely, if $\left\{f_{n}\right\}$ is increasing on one of these two intervals, then it is decreasing on the other. (If $\left\{f_{n}\right\}$ increases on both $(a, b)$ and $(b, a)$, then $f_{n+1}^{-1} f_{n}$ is not Möbius-like hyperbolic. See Figure 2.2.)

By replacing the $f_{n}$ 's by $f_{n}^{-1}$ 's, if necessary, we can assume that

$$
f_{1}>f_{2}>\ldots \text { on }(a, b), \quad f_{1}<f_{2}<\ldots \text { on }(b, a) .
$$




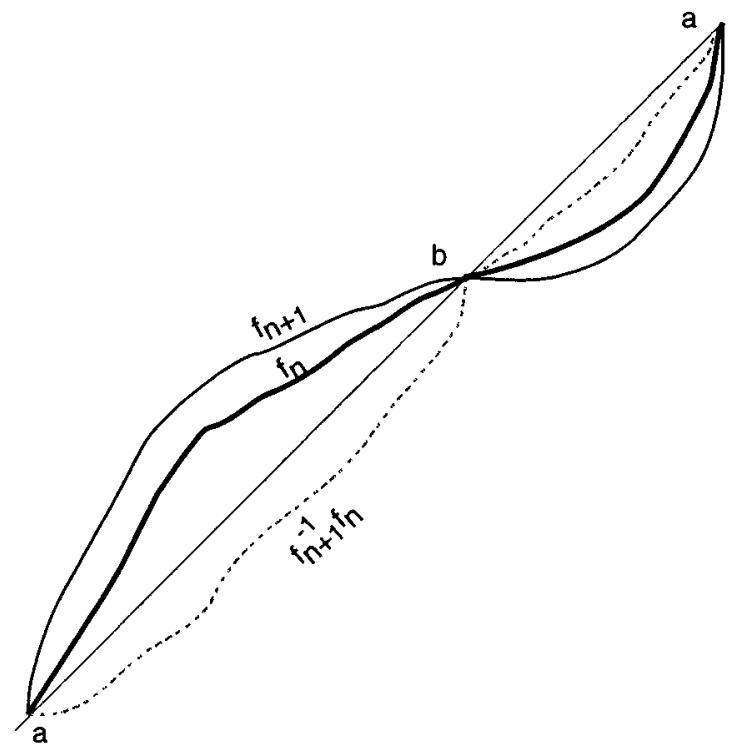

FiguRE 2.2

Also we can pass to a subsequence so that all $f_{n}$ 's share the same repulsive point and therefore share the same attractive point. Assume for example that $P_{f_{n}}=a, N_{f_{n}}=b$, for all $n$. See Figure 2.3.

Monotonicity of the sequences $\left\{f_{n}\right\}$ and $\left\{f_{n}^{-1}\right\}$ implies the existence of functions $f, f^{\prime}: S^{1} \rightarrow S^{1}$ so that

$$
f_{n} \rightarrow f, \quad f_{n}^{-1} \rightarrow f^{\prime} \quad \text { pointwise on } S^{1} .
$$

In order to show that $\left\{f_{n}\right\}$ satisfies the convergence property we only have to show that: $\left(^{*}\right)$ either both $f$ and $f^{\prime}$ are continuous (so they are homeomorphisms) or else both $f$ and $f^{\prime}$ are step functions consisting of jumps and flats of length 1.

Note that both $f$ and $f^{\prime}$ are nondecreasing functions (being limits of sequences of increasing functions), so the only type of discontinuity they can have is a jump. So assume $\left(^{*}\right)$ fails. That means that one of $f, f^{\prime}$ has a jump of length less than 1 at some point. Let us examine all the possibilities for such a jump and show that in each case one ends up with a contradiction.

1. $f$ cannot have a jump at $a$ since $P_{f_{n}}=a$ for all $n$. Similarly, $f^{\prime}$ has no jump at $b$.

2. $f$ cannot have any jumps on $(a, b) \cup(b, a)$ (and the same is true for $f^{\prime}$ ). Indeed, if $f$ had a jump at some $x \in(a, b)$, i.e., $f_{-}(x)<f_{+}(x)$ (see Figure 2.4), then by Lemma 2.2 we could find $g \in G$ such that $g(x) \in\left(f_{-}(x), f_{+}(x)\right)$. But then the graph of $g$ intersects the graph of $f_{n}$ for $n$ large enough, i.e., $g^{-1} f_{n}$ has more than two fixed points. Contradiction. Similarly for $x \in(b, a)$.

3. Finally, we show that $f$ cannot have a jump of length $<1$ at $b$ (similarly, $f^{\prime}$ has no jump of length $<1$ at $a$ ). Suppose the contrary, $f_{-}(b) \neq f_{+}(b)$. We distinguish the following cases:

- $f_{-}(b) \in(a, b)$ or $f_{+}(b) \in(b, a)$. See Figure 2.5. 


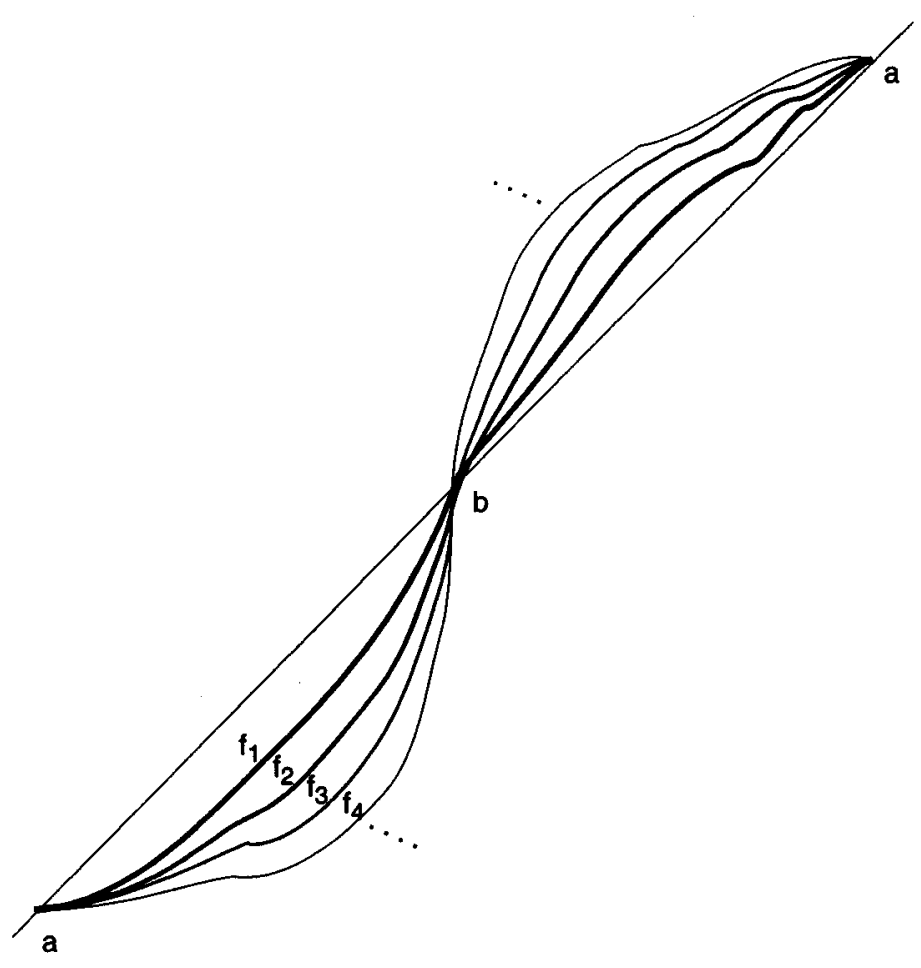

Figure 2.3

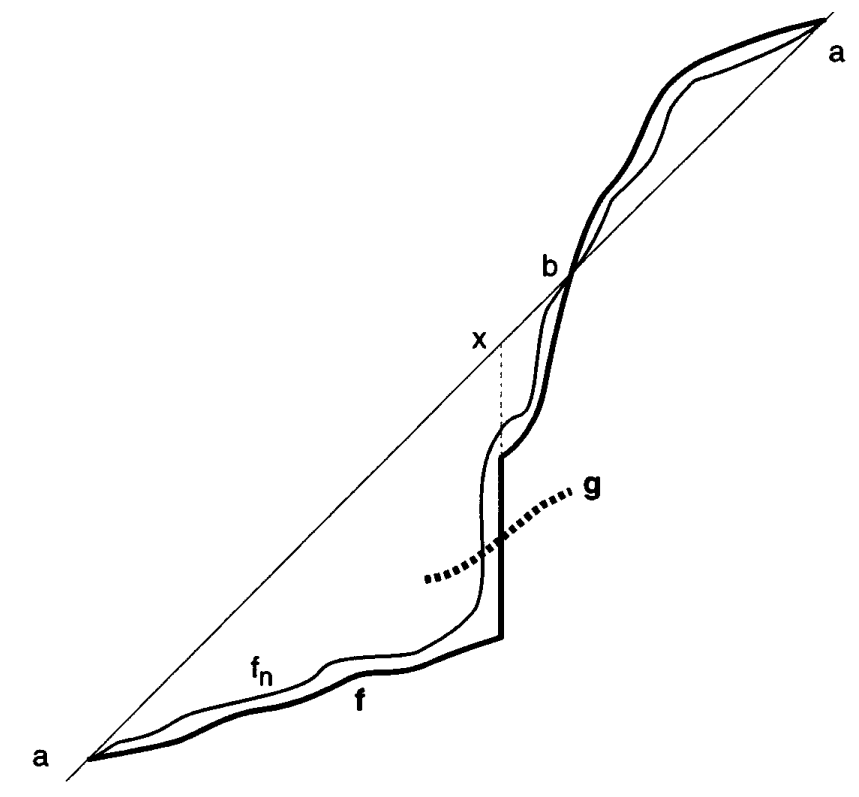

Figure 2.4 

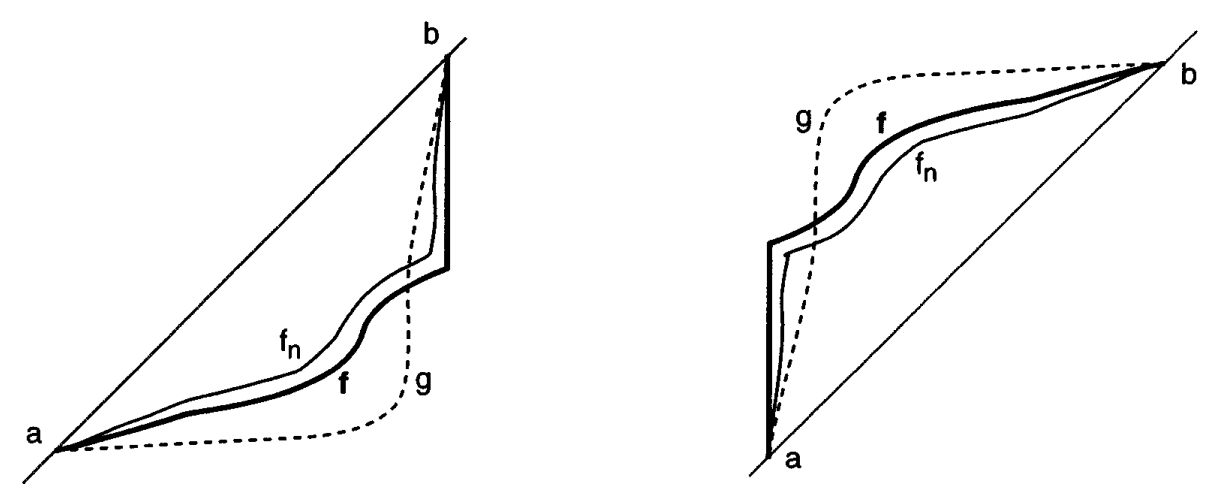

FiguRe 2.5

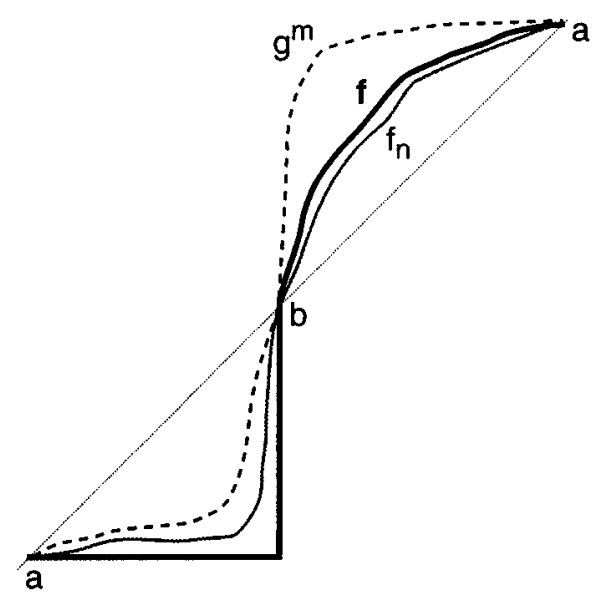

Figure 2.6

Choose any $g \in G$ with $P_{g}=a, N_{g}=b$. Then for $m$ large enough the graph of $g^{m}$ intersects the jump of $f$ at $b$ and hence the graphs of $f_{n}$ for $n$ large enough. Contradiction.

Similarly, we cannot have $f_{+}^{\prime}(a) \in(a, b)$ or $f_{-}^{\prime}(a) \in(b, a)$.

- $f_{-}(b)=a, f_{+}(b)=b \quad$ (The case $f_{-}(b)=b, f_{+}(b)=a$ is done similarly.) Refer to Figure 2.6.

Using 2 we conclude that $f$ is a homeomorphism on $[b, a]$. But then again for any $g \in G$ such that $P_{g}=a, N_{g}=b$ the element $g^{-m} f_{n}$ is not hyperbolic for $n, m$ large enough.

This finishes the proof under the assumptions that $P_{f_{n}}=a$ and $N_{f_{n}}=b$. Now we examine the case $P_{f_{n}}=b, N_{f_{n}}=a, \forall n$. Remember that our sequence $\left\{f_{n}\right\}$ satisfies the conditions $(\Delta)$. See Figure 2.7.

In the same way as before we obtain functions $f, f^{\prime}$. Clearly, $f$ cannot have jump at $b$ since $P_{f_{n}}=b$. Similarly, $f^{\prime}$ cannot have a jump at $a$. Now, the conditions $(\Delta)$ imply that $f$ cannot have a jump at $a$ either. Analogously, $f^{\prime}$ cannot have jump at $b$. So the only possibility for the jump of $f$ or $f^{\prime}$ of length $<1$ would be 


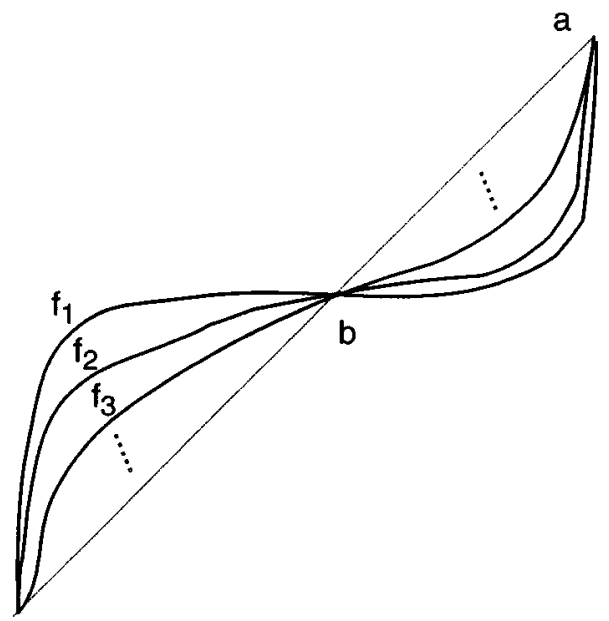

a

FiguRe 2.7
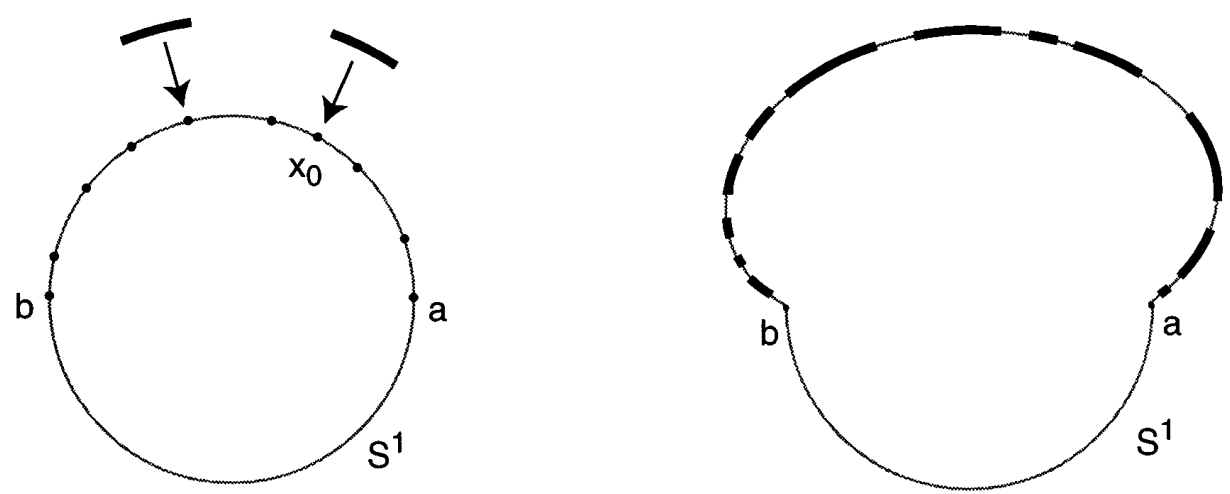

FiguRe 2.8

somewhere on $(a, b) \cup(b, a)$. But the same argument given in 2 works here as well. This finishes the proof that $G$ is a convergence group.

Remark 2.3. The hypothesis $L(G)=S^{1}$ cannot be dropped from the above theorem, as shown by the following example which is derived from Denjoy's construction. Take some countable nondiscrete purely hyperbolic Möbius group $G$, all of whose elements fix points $a$ and $b$ (e.g., the group generated by $x \mapsto \alpha x, x \mapsto \beta x$, with $\frac{\log \alpha}{\log \beta} \notin \mathbf{Q}$, where we view $S^{1}$ as $\boldsymbol{R} \cup\{\infty\}$ and $\left.a=0, b=\infty\right)$. Choose $x_{0} \in(a, b)$. Then, at every point in the $G$-orbit of $x_{0}$ insert a closed interval. Since $G$ is countable, there will be countably many such intervals, so we can make sure that the lengths of the inserted intervals when added up give a finite value. All this action takes place in $(a, b)$. See Figure 2.8. This process gives a new, bigger circle $\overline{S^{1}}$.

Now we can define an induced action of $G$ on $\overline{S^{1}}$ so that $G \hookrightarrow \mathrm{Homeo}_{+}\left(\overline{S^{1}}\right)$. Given $f \in G$, let $\bar{f}$ be a homeomorphism of $\overline{S^{1}}$ induced by $f$ in the following way. 


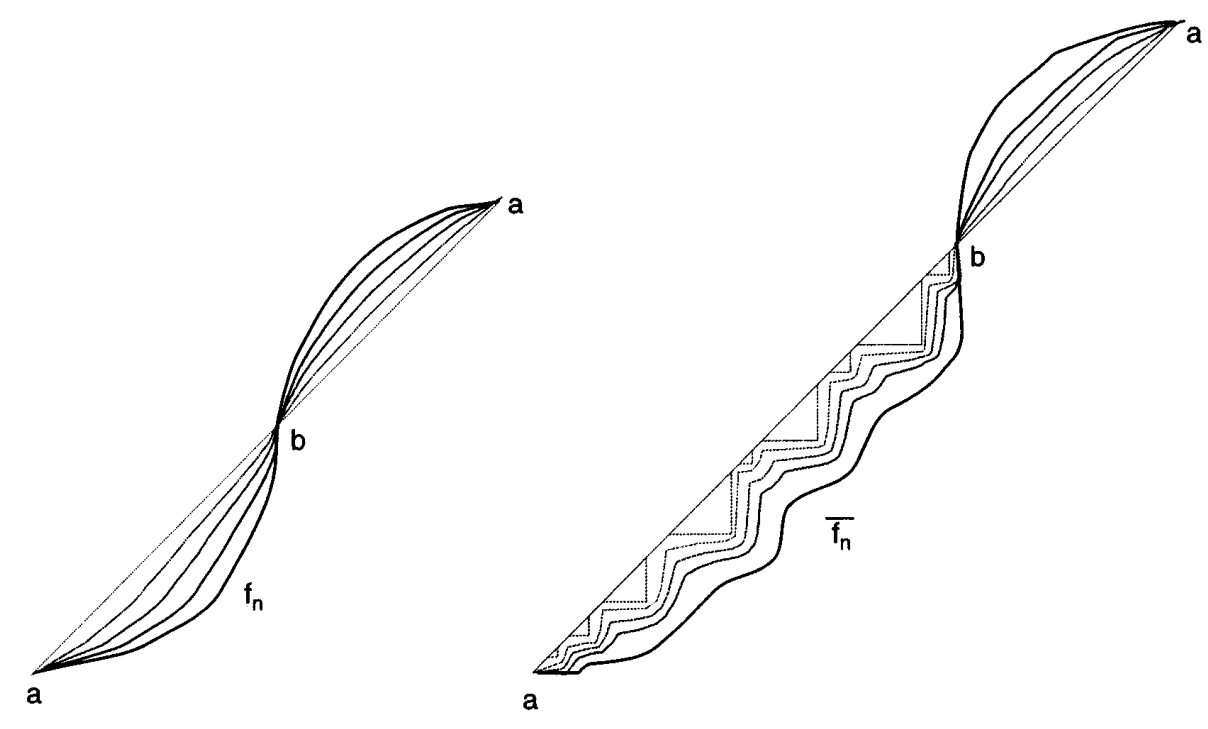

FiguRe 2.9

- If $x$ is a point untouched by the construction, i.e., no interval was inserted at $x$, set $\bar{f}(x)=f(x)$.

- Given an interval $[u, v]$ which was inserted at the point $z=h\left(x_{0}\right)$ for some $h \in G$, define $\bar{f}$ on $[u, v]$ to be the unique orientation preserving linear map which maps $[u, v]$ onto the interval inserted at $f(z)$.

Observe that the use of linear maps for extensions over the inserted intervals guarantees that $\overline{f_{1} \circ f_{2}}=\overline{f_{1}} \circ \overline{f_{2}}$ for any two $f_{1}, f_{2} \in G$. Set

$$
\bar{G}=\left\{i d \overline{S^{1}}\right\} \cup\left\{\bar{f} \mid f \in G-\left\{i d_{S^{1}}\right\}\right\} .
$$

The above observation implies that $\bar{G}$ is a group with the same group structure as $G$. Thus $\bar{G} \hookrightarrow \mathrm{Homeo}_{+}\left(\overline{S^{1}}\right)$ and every element of $\bar{G}$ is conjugate in $\mathrm{Homeo}\left(\overline{S^{1}}\right)$ to a Möbius transformation on $\overline{S^{1}}$. ${ }^{8}$ The group $\bar{G}$ preserves the union of the inserted intervals and thus $\bar{G}$ is not a convergence group acting on $\overline{S^{1}}$ : since $G$ is nondiscrete, we can find a sequence $\left\{f_{n}\right\}$ in $G$ converging to $i d_{S^{1}}$ and thus the induced sequence $\left\{\overline{f_{n}}\right\}$ in $\bar{G}$, having to preserve the union of the inserted intervals, does not satisfy the convergence property. See Figure 2.9.

Note. In the above example we disturbed the action of $G$ by inserting intervals at points of $(a, b)$ only. We could have defined a new circle $\overline{S^{1}}$ by inserting intervals at points of the orbit of $y_{0}$ for some $y_{0} \in(b, a)$ as well. The next theorem shows that the examples obtained in these two ways are essentially generic.

Theorem 2.4. Let $G \hookrightarrow \mathrm{Homeo}_{+}\left(S^{1}\right)$ be a non-cyclic group with two global fixed points $a$ and $b$, whose every element is Möbius-like. If $L(G) \neq S^{1}$, then $L(G)$ is either a Cantor set on $S^{1}$ or the union of two sets: the first being one of the two closed intervals on $S^{1}$ with endpoints $a, b$ and the second being a Cantor set

\footnotetext{
${ }^{8}$ Any homeomorphism of the circle that has two fixed points, one expanding and one contracting, is conjugate to a Möbius transformation. For a proof see [T].
} 
contained in the other interval with endpoints $a, b$. In any case $S^{1}-L(G)$ is an infinite union of disjoint open intervals $\left(x_{i}, y_{i}\right)$ so that $G$ with the induced action on a new circle $S_{\star}^{1}$, which is obtained from $S^{1}$ by identifying intervals $\left[x_{i}, y_{i}\right]$ to points, is a purely hyperbolic convergence group.

Proof of Theorem 2.4. Suppose $L(G) \neq S^{1}$. Note that $a, b \in L(G)$. We claim that $L(G)$ must have points in both $(a, b)$ and $(b, a)$. We will show that $L(G) \cap(a, b) \neq \emptyset$ and note that the same argument applies to $(b, a)$.

So suppose the contrary, that $L(G) \cap(a, b)=\emptyset$. Then the $G$-orbit of any $x \in(a, b)$ cannot accumulate on any point of $(a, b)$. Thus we can find $h \in G, h(x)>x$ which makes the distance between $x$ and $h(x)$ minimal. We will show that $G$ is generated by $h$, and thus reach a contradiction with the assumption that $G$ is non-cyclic. Indeed, given $g \in G, g \neq h, i d$ there exists an integer $n$ such that $h^{n+1}(x)>g(x)>h^{n}(x)$. Note that we cannot have $g(x)=h^{n}(x)$ since that would give three points of intersection between $h^{n}$ and $g$. Hence $h(x)>h^{-n} g(x)>x$ which contradicts the choice of $h$.

So $L(G)$ has points in both $(a, b)$ and $(b, a)$. We now want to prove that $L(G)$ is a perfect set, i.e., that it has no isolated points. Clearly, $a$ and $b$ are not isolated from either side. So assume that some point $x \in(a, b)$ is an isolated point of $L(G)$. Let $u, v$ be the points of $L(G)$ such that $u<x<v$ and $(u, x) \cap L(G)=$ $\emptyset,(x, v) \cap L(G)=\emptyset$. Clearly $\{u, v\} \cap\{a, b\}=\emptyset$. Now $x \in L(G)$ so there are infinitely many $g \in G$ such that $g(u, v) \cap(u, v) \neq \emptyset$. Clearly, for all these $g$ we must have $g(u, v) \propto(u, v)$. Even though for some of those $g$ we might have $g(u)$ or $g(v)$ equal $x$, the fact that there are infinitely many such $g$ guarantees that there will be many points of $L(G)$ in $[u, v]$ other than $x$. But this contradicts the choice of $u, v$. In the same way $L(G)$ has no isolated points in $(b, a)$. Thus $L(G)$ is a perfect set.

We now have two possibilities: the interior of $L(G)$ may be empty or not. Assume first that the interior of $L(G)$ is empty. Then, from the above it follows that $L(G)$ is a Cantor set on $S^{1}$, i.e.,

$$
S^{1}-L(G)=\bigcup_{i=1}^{\infty}\left(x_{i}, y_{i}\right)
$$

where $\left(x_{i}, y_{i}\right)$ are pairwise disjoint open intervals. Set

$$
S_{\star}^{1}=S^{1} /\left[x_{i}, y_{i}\right] \sim \text { point }, \quad i=1,2, \ldots .
$$

In other words, $S_{\star}^{1}$ is obtained from $S^{1}$ by identifying each closed interval $\left[x_{i}, y_{i}\right]$ to a point. Clearly, $S_{\star}^{1}$ is homeomorphic to $S^{1}$. Since $\bigcup_{i=1}^{\infty}\left[x_{i}, y_{i}\right]$ is invariant under the action of $G$, we conclude that there is an induced action of $G$ on $S_{\star}^{1}$. Write $G_{\star}$ when having this new action in mind. It is easy to see that $L\left(G_{\star}\right)=S_{\star}^{1}$, so by Theorem $2.1 G_{\star}$ is a convergence group.

Now if the interior of $L(G)$ is nonempty, then there exists a closed interval $[c, d] \subset L(G)$ which is a connected component of $L(G)$. Then $\{c, d\}=\{a, b\}$. Indeed, if say $c \in(a, b)$ then find a small positive $\varepsilon$ so that $(c, c+\varepsilon) \cap\{a, b\}=\emptyset$ and $(c, c+\varepsilon) \subset(c, d)$. Furthermore, $c+\varepsilon / 2 \in L(G)$ and we can think of $(c, c+\varepsilon)$ as a neighborhood of $\varepsilon / 2$, thus we can find $f \in G$ such that $f(c, c+\varepsilon) \sim(c, c+\varepsilon)$. Hence either $c \in f(c, c+\varepsilon)$ or $c \in f^{-1}(c, c+\varepsilon)$. In both cases we get a contradiction with the fact that $c$ is a limit point of $S^{1}-L(G)$. Without loss of generality we can assume $[c, d]=[a, b]$. Then $L(G)$ consists of $[a, b]$ and some subset of $[b, a]$ whose interior is empty by the latest argument. We showed earlier that $L(G)$ is 
a perfect set and thus $L(G) \cap[b, a]$ is a Cantor set. Therefore the action of $G$ on $[a, b]$ is Möbius. In the same way as above one can identify appropriate intervals in $[b, a]$ to points to obtain a new action $G_{\star}$ on a new circle (the action on $[a, b]$ stays unchanged). Then $G_{\star}$ is a convergence group.

\section{Groups With ONE GLOBAL FIXED POINT}

Theorem 3.1. Let $G \hookrightarrow H_{o m e o+}\left(S^{1}\right)$ be a group whose every element is Möbiuslike. Suppose $G$ has exactly one global fixed point a on $S^{1}$. In other words, every nontrivial element of $G$ is either parabolic fixing a, or hyperbolic with a being one of its fixed points. Assume also that $G$ is not cyclic. Then $G$ is a convergence group if and only if $L(G)=S^{1}$.

Lemma 3.2. If $L(G)=S^{1}$, then for every $x \in S^{1}-\{a\}$ the orbit of $x$ under $G$ is dense in $S^{1}$.

Proof of Lemma 3.2. Suppose there exists some $x \in S^{1}-\{a\}$ whose $G$-orbit $o(x)$ is not dense in $S^{1}$, i.e., $S^{1}-\overline{o(x)} \neq \emptyset$. The group $G$ does not have two global fixed points so $a \in \overline{o(x)}$. (To see this take any nontrivial $g \in G$ such that $g(x) \neq x$. If $g$ is parabolic, then $g^{n}(x) \rightarrow a$. If $g$ is hyperbolic, then either $g^{n}(x) \rightarrow a$ or $g^{-n}(x) \rightarrow a$.) Therefore we can find an open interval $(u, v) \subset S^{1}-\{a\}$ such that $(u, v) \subset S^{1}-\overline{o(x)}$ and $u, v \in \overline{o(x)}$.

The assumption $L(G)=S^{1}$ implies the existence of some $g \in G, g \neq i d$ such that $g(u, v) \cap(u, v) \neq \emptyset$. The set $S^{1}-\overline{o(x)}$ is $G$-invariant, so we cannot have $g(u, v) \propto(u, v)$. For the same reason neither $g(u, v) \subset(u, v)$ nor $g(u, v) \supset(u, v)$. Hence $g(u, v)=(u, v)$. But this gives $g$ at least three fixed points. Contradiction.

Proof of Theorem 3.1. $(\Leftarrow)$ : Suppose $G$ is a convergence group. Then $G$ is a Möbius group up to conjugation by some homeomorphism of $S^{1}$. We can assume that $G$ is a genuine Möbius group with one global fixed point. But every noncyclic Möbius group with one global fixed point is nondiscrete, so $L(G)=S^{1}$.

$(\Rightarrow)$ : Suppose $L(G)=S^{1}$. We distinguish two cases.

1. $G$ is a PURELY PARABOlic Group, i.e., every element of $G$ is parabolic. The graphs of lifts to $\boldsymbol{R}$ of distinct elements of $G$ are disjoint everywhere except at where they all intersect, so this case can be done in much the same way as in the proof of Theorem 2.1.

Namely, we start with an arbitrary sequence $\left\{f_{n}\right\}$ in $G$. After passing to a subsequence we can assume that $\left\{f_{n}\right\}$ is monotonic. By replacing $f_{n}$ 's by $f_{n}^{-1}$ 's if necessary, we can assume that $\left\{f_{n}\right\}$ is increasing. Hence there exist limit functions $f, f^{\prime}: S^{1} \rightarrow S^{1}$ such that

$$
f_{n} \rightarrow f, f_{n}^{-1} \rightarrow f^{\prime} \text { pointwise on } S^{1} .
$$

Also, we can pass to a subsequence so that all $f_{n}$ 's move the points of $S^{1}-\{a\}$ in the same direction. First assume that all $f_{n}$ 's move the points of $S^{1}-\{a\}$ in the counterclockwise direction. See Figure 3.1.

As before, in order to prove that $\left\{f_{n}\right\}$ satisfies the convergence property we only need to show either both $f$ and $f^{\prime}$ are continuous (so they are homeomorphisms) or else both $f$ and $f^{\prime}$ are step functions consisting of jumps and flats of length 1 .

So assume the contrary, that say $f$ has a jump discontinuity of length less than 1. Figure 3.2 shows that such a jump cannot occur at the point $a$. Namely, we can take any parabolic $g \in G$ moving points of $S^{1}-\{a\}$ in the counterclockwise 


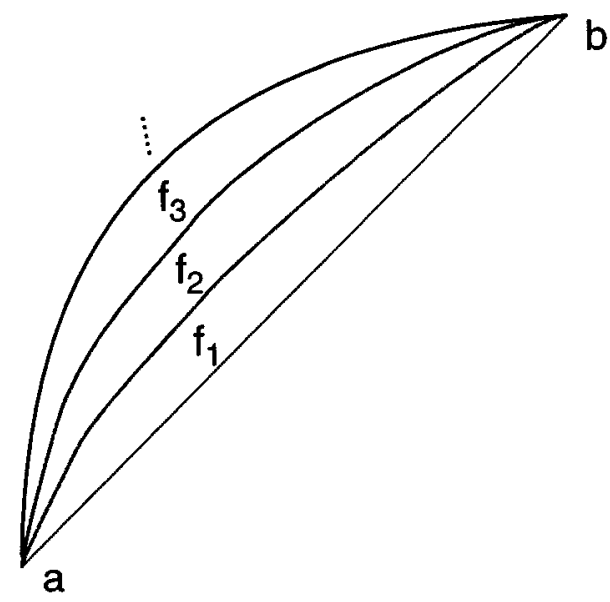

Figure 3.1

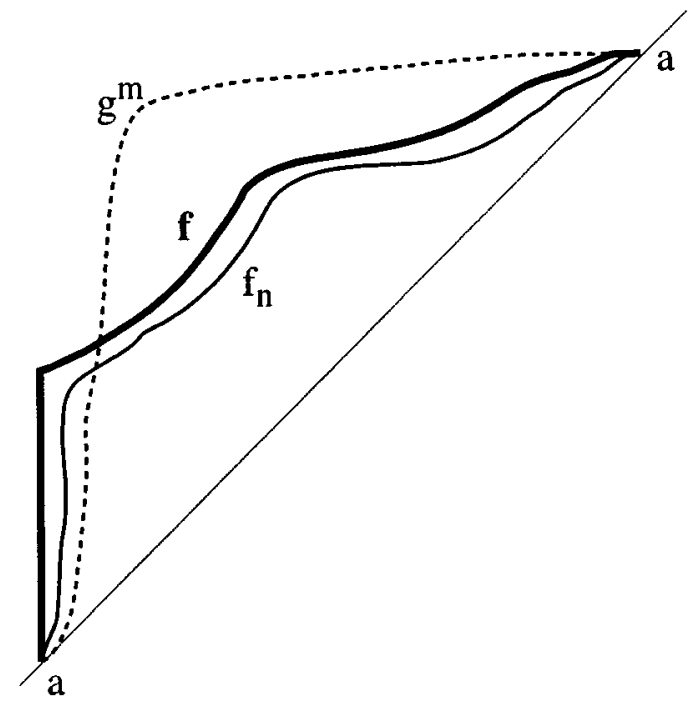

FiguRE 3.2

direction. Then, for $m$ large enough the graph of $g^{m}$ intersects the graph of $f$ at some point other than $a$ and hence it also intersects the graph of $f_{n}$ at some point other than $a$, for some large index $n$. But this contradicts our assumption that $G$ is a purely parabolic group.

So now we only need to show that $f$ cannot have a jump of length less than 1 at some point other than $a$ either. If such a jump existed at some point $x$, then by Lemma 3.2 we could find some $g \in G$ such that $g(x) \in\left(f_{-}(x), f_{+}(x)\right)$. But then the graph of $g$ would have to intersect the graph of some $f_{n}$ with high index $n$ somewhere near $x$. That contradicts the fact that $G$ is purely parabolic. This finishes the proof under the assumption that the $f_{n}$ 's move the points of $S^{1}-\{a\}$ in the counterclockwise direction. 


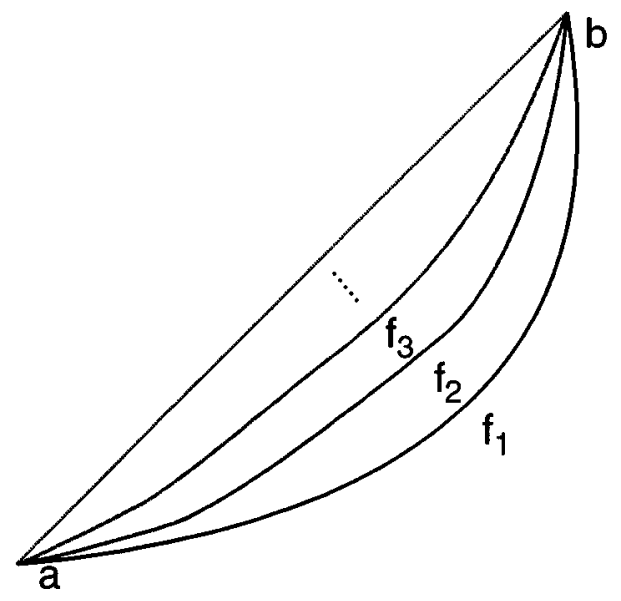

FiguRE 3.3

Now assume that the $f_{n}$ 's move the points of $S^{1}-\{a\}$ in the clockwise direction. Recall that $\left\{f_{n}\right\}$ is increasing on $(a, a)$. See Figure 3.3. We obtain functions $f$ and $f^{\prime}$ in the same way as above.

Again assume that say $f$ has a jump discontinuity of length less than 1 . Under the assumptions we made it is obvious that $f$ cannot have any kind of jump at $a$. So a jump of length less than 1 has to occur at some point $x \in(a, a)$. Then the same argument as above shows that this leads to a contradiction. This finishes the proof in the case when $G$ is a purely parabolic group.

2. $G$ Has hyperbolic elements. By Lemma 3.2 it follows that $L_{0}$, the set of fixed points of all hyperbolic elements of $G$, is dense in $S^{1}$.

Let $\left\{f_{n}\right\}$ be a sequence of distinct elements in $G$. We want to show that $\left\{f_{n}\right\}$ satisfies the convergence property.

First we show that after passing to a subsequence, we can get all the difference functions $f_{m}^{-1} f_{n}$ 's with $m>n$, to be of the same type, i.e., all parabolic or all hyperbolic. This can be done in purely combinatorial way. To $\left\{f_{n}\right\}$ we can associate the following "matrix of relations":

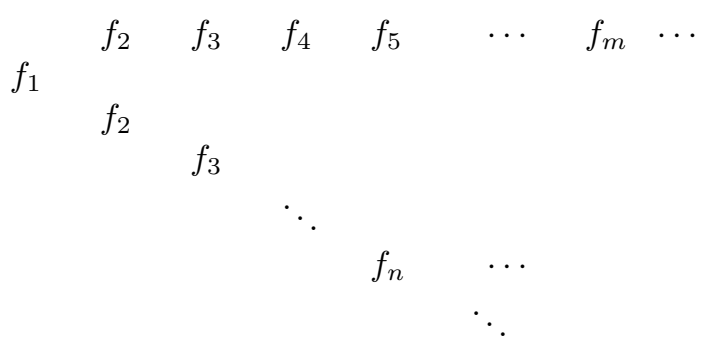

where at the intersection of $n$-th row and $m$-th column we put 1 if $f_{m}^{-1} f_{n}$ is parabolic, and we put 2 if $f_{m}^{-1} f_{n}$ is hyperbolic (numbers 1 and 2 correspond to the number of intersection points between the graphs of $f_{n}$ and $f_{m}$ ). 
Combinatorial claim. ${ }^{9}$ There exists a subsequence of $\left\{f_{n}\right\}$ so that the matrix corresponding to that subsequence consists of either 1's only, or 2's only.

Proof of the combinatorial claim. Label every row of the matrix corresponding to $\left\{f_{n}\right\}$ by 1,2 or 12 according to the following rule:

label 1 if the row contains only finitely many 2's,

label 2 if the row contains only finitely many 1's,

label 12 if the row contains infinitely many of both 1's and 2's.

Special case. Suppose that the matrix corresponding to $\left\{f_{n}\right\}$ has infinitely many rows labeled $[1$. Then we can pass to a subsequence so that its matrix consists of 1 's only.

Proof of the special case. Since there are infinitely many rows labeled 1, we can first pass to a subsequence consisting of elements corresponding to these rows, i.e., we are getting rid of rows which are not labeled 1 . So now we have a new infinite sequence, call it $\left\{f_{n}\right\}$ again, whose matrix has the property that all its rows are labeled 1 . Set $f_{n_{1}}$ to be the first element of this sequence. Then get rid of finitely many elements of $\left\{f_{n}\right\}$ which are producing 2's in the first row of the matrix of $\left\{f_{n}\right\}$. Let $f_{n_{2}}$ be the next element among those that are left. Continue ... get rid of finitely many elements that produce 2's in the second row (note that the first row has 1's only), and take $f_{n_{3}}$ to be the next among those that are left ... The sequence $\left\{f_{n_{i}}\right\}$ has the required property. This finishes the proof in the special case.

Now in general, given $\left\{f_{n}\right\}$ consider its matrix. If it contains infinitely many rows labeled 1 or infinitely many rows labeled 2 , we are done by the special case. So assume all rows except finitely many are labeled 12 . Get rid of those finitely many elements whose rows are not labeled 12 . Now we have a sequence whose matrix has all rows labeled 12 .

After passing to a subsequence we can get the first row to consist of 1's only. Let $f_{n_{1}}$ be the first element of this subsequence. Note that when we passed to this subsequence we obtained a new matrix with new labelings for the second, third, ... rows. This matrix can either have infinitely many rows labeled 1 , or infinitely many rows labeled 2 , or it can have all but finitely many rows labeled 12 . The first two possibilities put us in the special case, so we can assume that (after getting rid of finitely many elements) our matrix has all rows starting with the second one (remember the first row consists of 1's only) labeled 12 . Let $f_{n_{2}}$ be the element corresponding to the second row of this last matrix. As before, throw out infinitely many elements of the sequence which produced 2's in the second row (i.e., pass to a subsequence). Now both the first and second row consist of 1's only, but the rest of the rows have new assignments. Again we are either done by the special case, or else we can continue our process and define $f_{n_{3}} \ldots$. If this process ever stops, we are done by the special case, and if it never stops, then it gives us an infinite subsequence $\left\{f_{n_{i}}\right\}$ with the required property.

\section{End of proof of the combinatorial claim.}

\footnotetext{
${ }^{9}$ This combinatorial claim is in fact a case of so-called Ramsey's theorem. See $[R]$.
} 


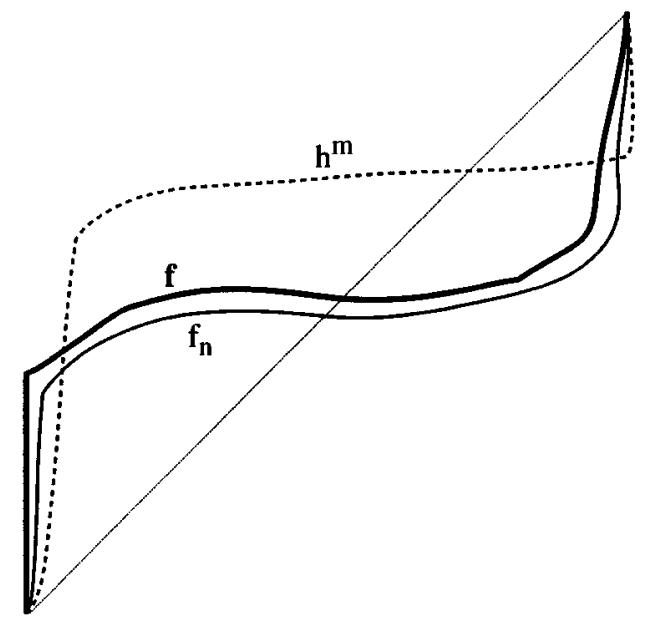

FigURE 3.4

Now back to our initial sequence $\left\{f_{n}\right\}$. As shown above, after passing to a subsequence, we can assume that all $f_{m}^{-1} f_{n}$ 's are of the same type.

Case II.1: $\forall m>n, f_{m}^{-1} f_{n}$ is parabolic. In other words, the graphs of the $f_{n}$ 's are pairwise disjoint everywhere except at the point $a$ where they all intersect. Therefore, after passing to a subsequence, $\left\{f_{n}\right\}$ is totally ordered, say $f_{1}<f_{2}<\cdots$ on $(a, a)$. So there exist functions $f, f^{\prime}: S^{1} \rightarrow S^{1}$ such that $f_{n} \rightarrow f$ and $f_{n}^{-1} \rightarrow f^{\prime}$ pointwise.

Suppose first that $f$ has a jump of length less than 1 at some point. Figure 3.4 shows that such a jump cannot occur at $a$. Namely, one first notices the fact that $\left\{f_{n}\right\}$ is increasing on $(a, a)$ and that $f_{n}(a)=a, \forall n$ imply $f_{-}(a)=a$. So, if $f_{+}(a)>a$, then choose any hyperbolic $h \in G$ such that $N_{h}=a, P_{h} \in\left(f_{+}(a), a\right)$. Clearly, for $m$ large enough the graph of $h^{m}$ will intersect the graph of $f$ more than twice. Consequently, for $n$ large enough $f_{n}^{-1} h^{m}$ has more than two fixed points. Contradiction.

So now assume a jump of length less than 1 occurs at some point $x \in(a, a)$. Denote $J=\left(f_{-}(x), f_{+}(x)\right)$.

Claim. (Simplicity condition). There is no $h \in G$ such that $h(J) \curvearrowright J$.

Proof of claim. If such $h$ exists, then by replacing $h$ by $h^{-1}$, if necessary, we can assume that $h$ moves $J$ in the counterclockwise direction. Form a new sequence $\left\{h f_{n}\right\}$ and denote $h f=\lim _{n \rightarrow \infty} h f_{n}$. Then

$$
(h f)_{-}(x)=h\left(f_{-}(x)\right), \quad(h f)_{+}(x)=h\left(f_{+}(x)\right) .
$$

In other words, $h f$ has a jump $h(J)$ at $x$. See Figure 3.5. Choose some high index $n$ so that the graph of $h f_{n}$ intersects the graph of $f$ twice near $x$. Then for $m$ large enough the graph of $h f_{n}$ intersects twice the graph of $f_{m}$ near $x$. This together with the global fixed point $a$ gives three intersection points. A contradiction.

Now we will finish the proof by contradiction; i.e., we will prove that $h$ as in the claim really exists, for any interval $J=(u, v)$ not containing $a$. Let $p$ be any parabolic element of $G$ which moves points of $S^{1}-\{a\}$ in a clockwise direction. 

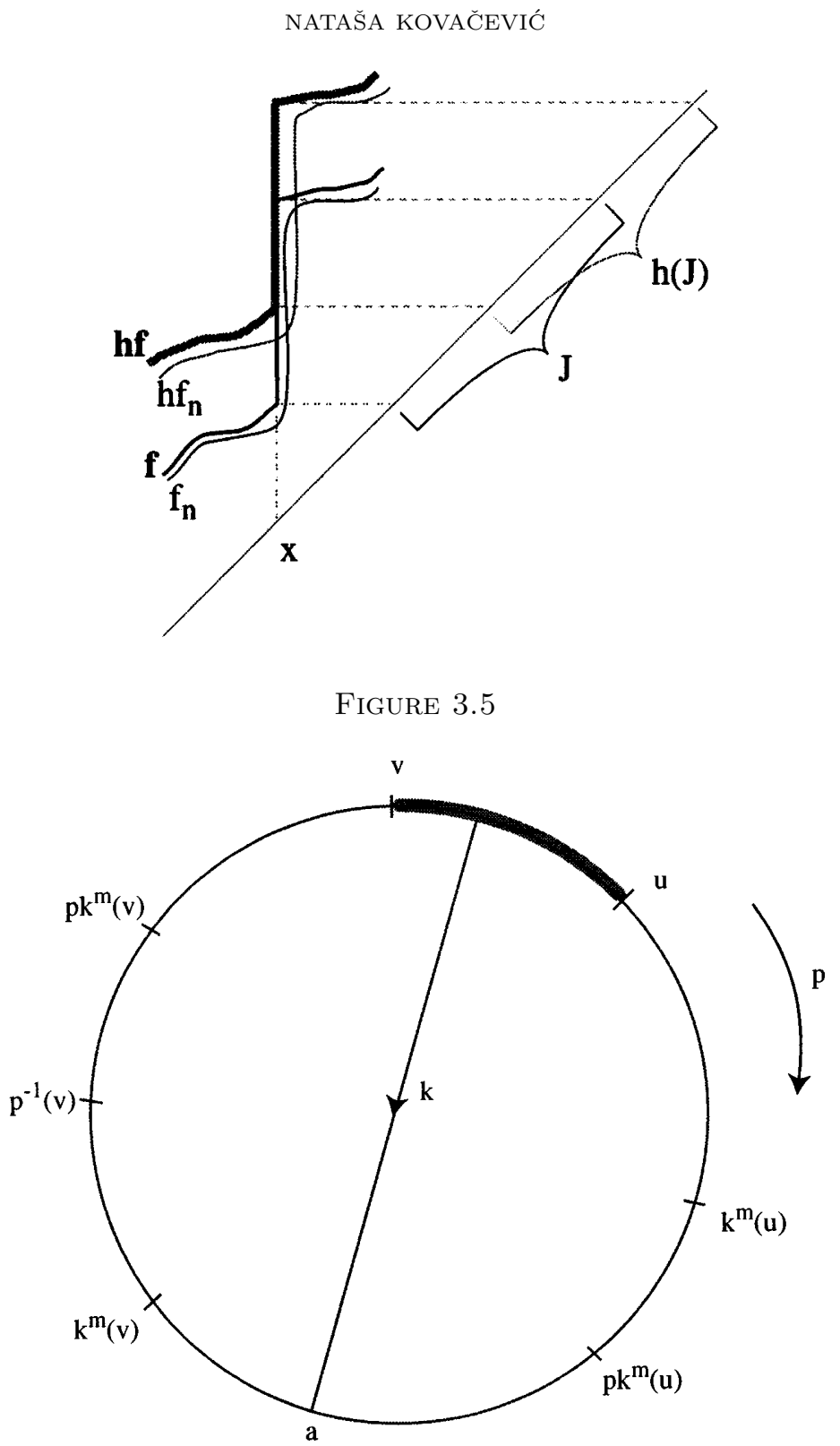

FiguRE 3.6

Refer to Figure 3.6. Let $k \in G$ be a hyperbolic element such that $N_{k} \in(u, v)$. Choose a large enough power $m$ of $k$ so that

$$
k^{m}(v)>p^{-1}(v)>v .
$$

Then $k^{m}(v)>p k^{m}(v)>v$, while $p k^{m}(u)<k^{m}(u)$. Hence $k^{m}(u, v) \curvearrowright p k^{m}(u, v)$. In other words,

$$
(u, v) \nsim k^{-m} p k^{m}(u, v),
$$

so we can put $h=k^{-m} p k^{m}$. 


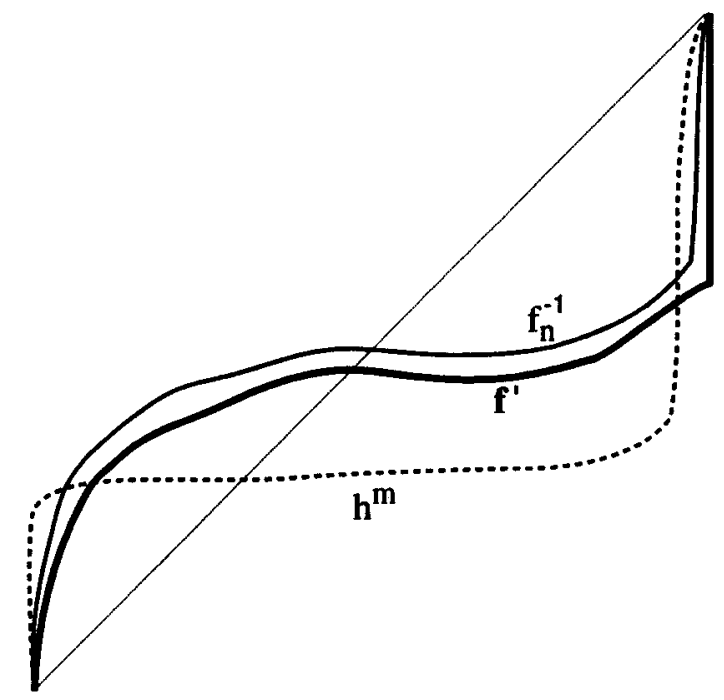

FiguRE 3.7

So far we have proved that $f$ cannot have jump discontinuities of length less than 1. Now, in order to prove the same for $f^{\prime}$, we first note that $f^{\prime}$ cannot have such jumps at points of $(a, a)$ by the same argument which we used for $f$. So we only need to check whether $f^{\prime}$ can have a jump of length less than one at $a$. Recall that our initial assumption is that the sequence $\left\{f_{n}\right\}$ is increasing, i.e., $\left\{f_{n}^{-1}\right\}$ is decreasing. Hence $f_{+}^{\prime}(a)=a$. Refer to Figure 3.7. If $f_{-}^{\prime}(a) \in(a, a)$, then choose any hyperbolic $h \in G$ such that $N_{h}=a, P_{h} \in\left(a, f_{-}^{\prime}(a)\right)$. Clearly, for $m$ large enough the graph of $h^{m}$ will intersect the graph of $f^{\prime}$ more than twice. Contradiction.

Thus we have proved that the arbitrary sequence $\left\{f_{n}\right\}$ in which all the difference functions $f_{m}^{-1} f_{n}$ with $m>n$ are parabolic satisfies the convergence property.

Case II.2: $\quad \forall m>n, f_{m}^{-1} f_{n}$ is hyperbolic. For every two elements $f_{m}$ and $f_{n}$, $m>n$, either $P_{f_{m}^{-1} f_{n}}=a$ or $N_{f_{m}^{-1} f_{n}}=a$. By the same combinatorial argument as before (only here the "matrix of relations" has either $P$ or $N$ at the intersection of the $n$-th row and the $m$-th column, depending on whether $P_{f_{m}^{-1} f_{n}}=a$ or $N_{f_{m}^{-1} f_{n}}=a$ ) we can assume that, after passing to a subsequence and then replacing $\left\{f_{n}\right\}$ by $\left\{f_{n}^{-1}\right\}$ if necessary, we have $P_{f_{m}^{-1} f_{n}}=a, \forall m>n$.

We now do some further refining of $\left\{f_{n}\right\}$. First consider the sequence of points $\left\{N_{f_{n}^{-1} f_{1}}\right\}$ for $n>1$. We can replace the sequence $\left\{f_{n}\right\}, n \geq 2$, by its subsequence, which we call $\left\{f_{n}\right\}$ again, so that $\left\{N_{f_{n}^{-1} f_{1}}\right\}$ converges monotonically to some point $b_{1}$. Now consider $\left\{N_{f_{n}^{-1} f_{2}}\right\}$ for $n>2$. Then do the same kind of renaming so that afterwards $\left\{N_{f_{n}^{-1} f_{2}}\right\}$ converges to some point $b_{2}$. Continue this process to obtain sequence $\left\{b_{n}\right\}$. As a result, our sequence $\left\{f_{n}\right\}$ has the property that for each $n$

$$
N_{f_{m}^{-1} f_{n}} \rightarrow b_{n}, m>n, m \rightarrow \infty .
$$

Once this is established, we could pass to some subsequence of $\left\{f_{n}\right\}$, rename it $\left\{f_{n}\right\}$, rename $b_{n}$ 's accordingly, and still have property (o) intact. The sequence $\left\{b_{n}\right\}$ can be made to converge monotonically to some point $b$ by passing to a subsequence. 


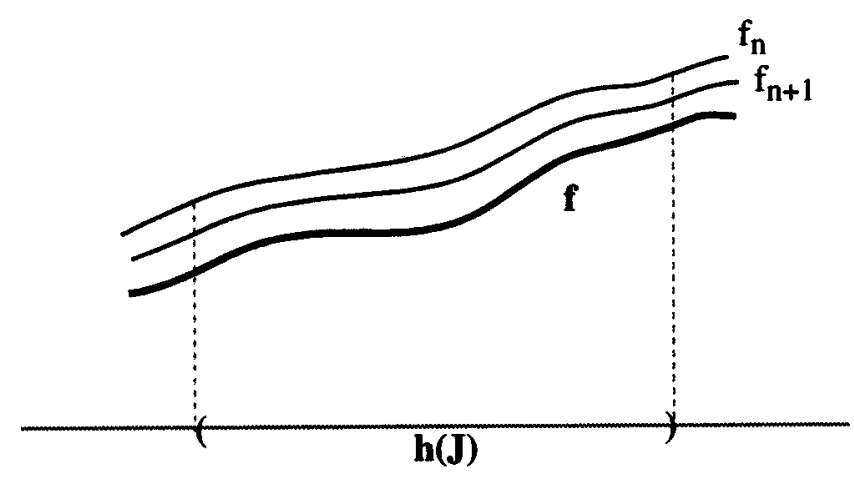

FiguRe 3.8

In view of what was said above, after passing to a corresponding subsequence of $\left\{f_{n}\right\}$, property (o) still holds.

(i) First assume $b \neq a$.

At this point our sequence $\left\{f_{n}\right\}$ is pretty "tame", i.e., it has the following property (after a subsequence if necessary): given any $\varepsilon>0$, there exists a high enough index $n$ so that

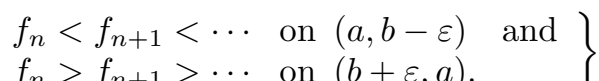

In other words, $\forall x \in S^{1}$ the sequence $\left\{f_{n}(x)\right\}$ becomes strictly monotonic after a large enough index, and the same is true for $\left\{f_{n}^{-1}(x)\right\}$. Therefore, there exist functions $f, f^{\prime}: S^{1} \rightarrow S^{1}$ such that $f_{n} \rightarrow f$ and $f_{n}^{-1} \rightarrow f^{\prime}$ pointwise on $S^{1}$.

By the same arguments as in II.1, one can show that neither $f$ nor $f^{\prime}$ can have a jump of length $<1$ at $a$.

So let us show that $f$ cannot have a jump of length $<1$ at some $x \in S^{1}-\{a, b\}$. Once that is established, we will only have to show that a jump of length less than 1 cannot occur at $b$. Suppose the contrary, that we have some $x \in(a, b) \cup(b, a)$ such that $f_{-}(x) \neq f_{+}(x)$. Set $J=\left(f_{-}(x), f_{+}(x)\right)$. Note that $a \notin \bar{J}$; otherwise $f^{\prime}$ has a jump of length less than 1 at $a$.

We now list some consequences of the assumptions that we have made so far.

Claim 1 (Simplicity condition). $h(J) \curvearrowright J$ for no $h \in G$.

Proof of Claim 1. The same as in II.1.

Claim 2. For every $h \in G$ such that $b \notin \overline{h(J)}, f$ is flat on $h(J)$.

Proof of Claim 2. If not, then $f$ is non-constant on $h(J)$ so for large enough $n$ we get $f_{n}(h(J)) \curvearrowright f_{n+1}(h(J))$, i.e., $h^{-1} f_{n+1}^{-1} f_{n} h(J) \curvearrowright J$. See Figure 3.8. Contradiction with Claim 1.

Set $A=\left\{y \in S^{1} \mid f\right.$ is flat in some neighborhood of $\left.y\right\}$.

Claim 3. $L_{0}-\{a, b\} \subset A .{ }^{10}$

\footnotetext{
${ }^{10}$ Recall that $L_{0}$ denotes the set of all fixed points of hyperbolic elements of $G$.
} 


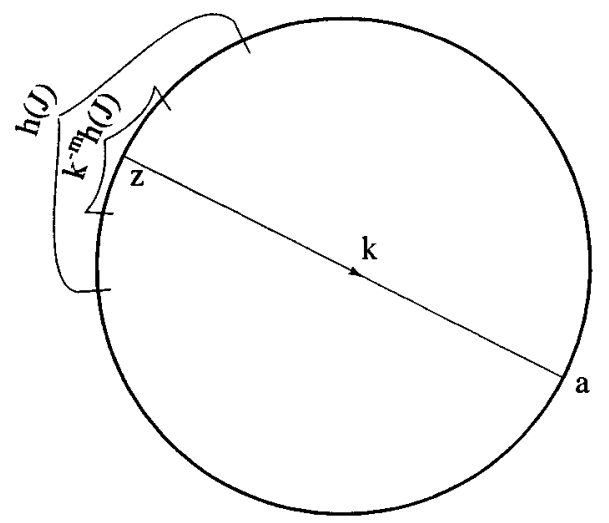

FiguRe 3.9

Proof of Claim 3. Suppose $z \in L_{0}-\{a, b\}$, i.e., $z=N_{k}, a=P_{k}$ for some hyperbolic element $k$ of $G$. By Lemma 3.1, the $G$-orbit of $z$ is dense in $S^{1}$, so $z \in h(J)$ for some $h \in G$. For sufficiently large $m$, the interval $I=k^{-m}(h(J))$ is small enough so that its closure contains neither $a$ nor $b$. See Figure 3.9. By Claim $2 f$ is flat on $I$ and $z \in I$, so $z \in A$.

As noted before $L_{0}$ is dense in $S^{1}$, so $A$ is open and dense, and thus $S^{1}-A$ is a closed nowhere dense set on $S^{1}$ containing $a$. Let $c$ be a point in $S^{1}-A$ so that $b<c<a$. Note first that $c$ must be a limit point of $S^{1}-A$. Indeed, if on the contrary $c$ were an isolated point of $S^{1}-A$, that would imply the existence of two flats of $f$, one to the left, denoted $I$, and one to the right of $c$, and a jump at $c$. Thus, for $n$ large enough we would have $f_{n}(I) \curvearrowright f_{n+1}(I)$, which would contradict the simplicity condition (only here applied to $I$ as a jump of $f^{\prime}$ ). Thus $c$ is a limit point of $S^{1}-A$. The same is true for all points of $S^{1}-A$ lying in $(c, a)$, and so $[c, a] \cap\left(S^{1}-A\right)$ is a Cantor set. From now on we shall concentrate exclusively on the interval $(c, a)$. Write

$$
(c, a) \cap A=\bigcup_{i=1}^{\infty}\left(x_{i}, y_{i}\right),
$$

where $\left(x_{i}, y_{i}\right)$ are disjoint open intervals. From the definition of the intervals $\left(x_{i}, y_{i}\right)$ it is clear that each one of them represents a maximal interval of flatness of the limit function $f$ in the sense that if $f$ is flat on an open interval $I \subset(c, a)$, then $I \subset\left(x_{i}, y_{i}\right)$ for some $i$.

Claim 4. $h\left(x_{i}, y_{i}\right) \nsim\left(x_{i}, y_{i}\right)$ for no $h \in G$ and no $i$.

Proof of Claim 4. Since $\left\{f_{n}\right\}$ is monotonically decreasing on $(c, a)$ (equivalently, $\left\{f_{n}^{-1}\right\}$ is monotonically increasing on $\left.(c, a)\right)$, and each $\left(x_{i}, y_{i}\right)$ can be viewed as a jump of $f^{\prime}$, this claim is true for the same reason as Claim 1.

Claim 5. If $h, k \in G$ are hyperbolic with $N_{h}, N_{k} \in\left(x_{i}, y_{i}\right)$, then $h$ and $k$ intersect within $\left(x_{i}, y_{i}\right)$, i.e., $h^{-1} k$ is hyperbolic with one fixed point in $\left(x_{i}, y_{i}\right)$.

Proof of Claim 5. If not, then $h\left(x_{i}, y_{i}\right) \sim k\left(x_{i}, y_{i}\right)$. See Figure 3.10. Equivalently, $k^{-1} h\left(x_{i}, y_{i}\right) \curvearrowright\left(x_{i}, y_{i}\right)$ which contradicts Claim 4.

Claim 6. $f$ is continuous at every $y_{i}, i=1,2, \ldots$ 


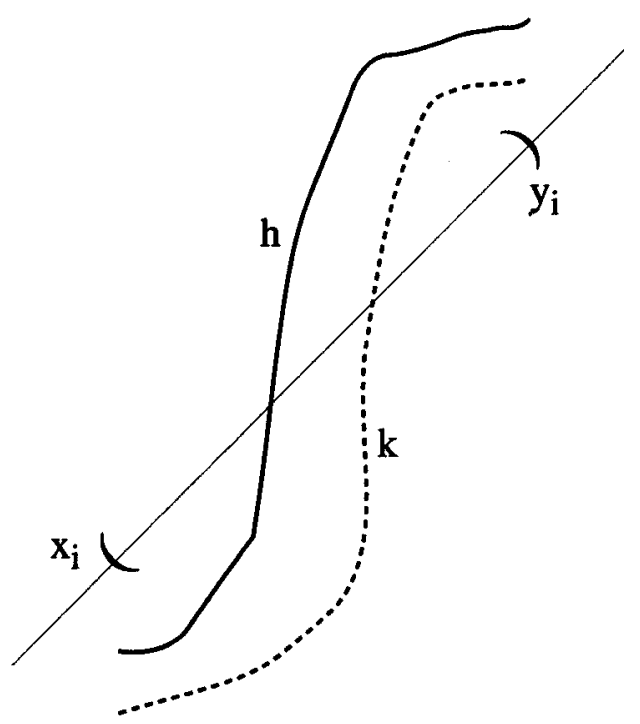

FiguRe 3.10

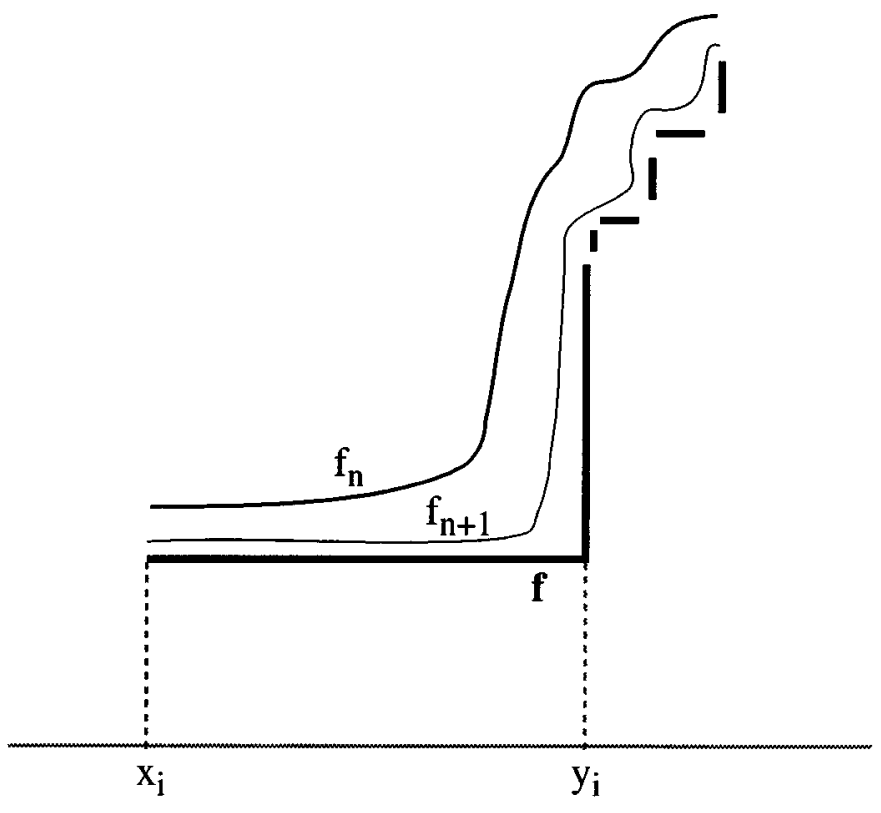

FiguRe 3.11

Proof of Claim 6. If not, then $f$ has a jump at $y_{i}$, so $f$ is not constant on $\left[x_{i}, y_{i}\right]$, that is, $f\left(x_{i}\right)<f\left(y_{i}\right)$. Thus for $n$ large enough $f_{n}\left(x_{i}, y_{i}\right) \propto f_{n+1}\left(x_{i}, y_{i}\right)$. See Figure 3.11. Contradiction to Claim 4.

Claim 7. Let $g_{n}=f_{n+1}^{-1} f_{n}$. Then the sequence $\left\{g_{n}\right\}$, when considered on the interval $(c, a)$, converges to a function $g$ whose graph consists of triangles arranged 


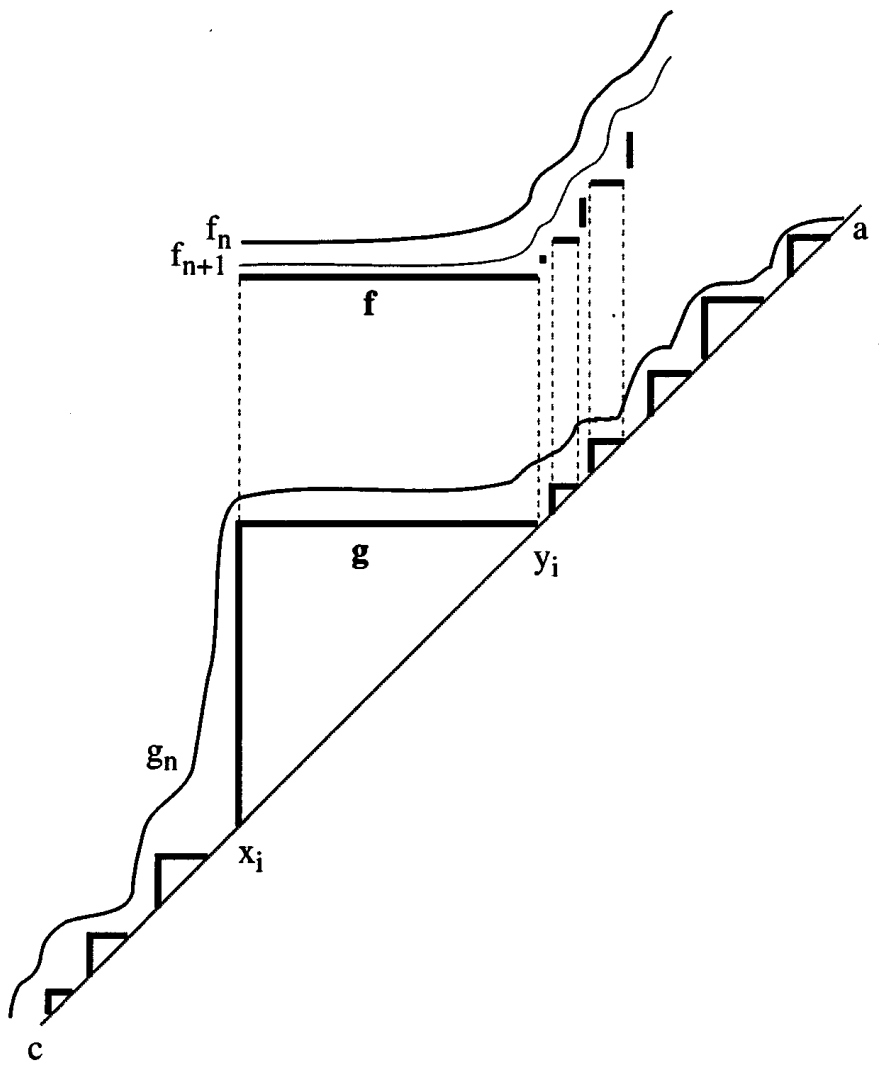

FiguRe 3.12

along the diagonal $y=x$, which are determined by the intervals $\left(x_{i}, y_{i}\right)$ and their limit points. See Figure 3.12. More precisely,

$$
\lim _{n \rightarrow \infty} g_{n}(x)=y_{i}, \quad x \in\left[x_{i}, y_{i}\right], \quad i=1,2, \ldots .
$$

Moreover, one can pass to a subsequence of $\left\{g_{n}\right\}$ and rename it $\left\{g_{n}\right\}$, so that the sequence $\left\{g_{n}\right\}$ converges to $g$ on $(c, a)$ in a strictly decreasing manner.

Proof of Claim 7. Fix $i$ and let us see how does the sequence $g_{n}$ behave on $\left(x_{i}, y_{i}\right)$. Recall that the $f_{n}$ 's converge to $f$ in a decreasing manner, $f$ is flat on $\left(x_{i}, y_{i}\right)$ and continuous at $y_{i}$. See Figure 3.12. So $f_{n+1}^{-1} f_{n}=g_{n}$ is an increasing function on $\left(x_{i}, y_{i}\right)$ (actually, on all of $(c, a)$ ). This, together with Claim 4, implies that $x_{i}<y_{i} \leq g_{n}\left(x_{i}\right)<g_{n}\left(y_{i}\right)$. Moreover, continuity of $f$ at $y_{i}$ implies that as the index $n$ increases, the intervals $g_{n}\left(x_{i}, y_{i}\right)$ become very small and very close to $y_{i}$. Therefore $g_{n} \rightarrow y_{i}$ on $\left[x_{i}, y_{i}\right]$. Since $i$ was chosen arbitrarily, this proves that the limit function $g$ has the right behavior.

Now fix $i$ again and pass to a subsequence so that $\left\{g_{n}\right\}$ is decreasing on $\left(x_{i}, y_{i}\right)$. We claim that $\left\{g_{n}\right\}$ is then decreasing on all of $(c, a)$, after a high enough index. Indeed, if this is not the case, then for some arbitrarily large $n, g_{n}$ and $g_{n+1}$ intersect at some point $z \in(c, a)$. Let $t$ be $g_{n+1}^{-1} g_{n}$ or $g_{n}{ }^{-1} g_{n+1}$ depending on whether $z$ comes before or after $\left(x_{i}, y_{i}\right)$. In any case $z \in L_{0} \subset A-\{a, b\}$, so $z$ is contained in some 


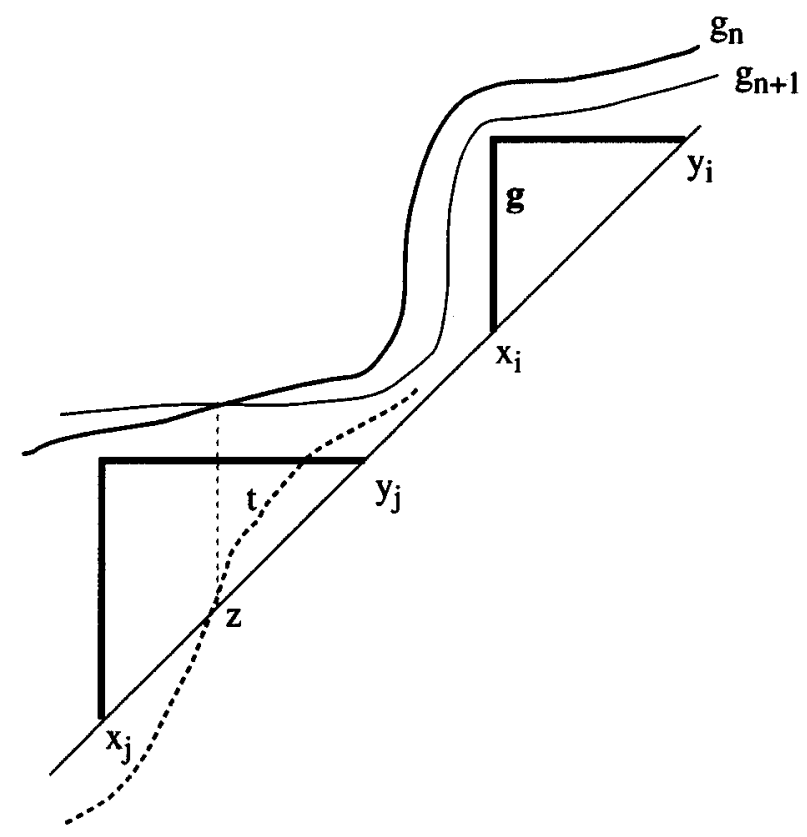

FigURE 3.13

$\left(x_{j}, y_{j}\right)$. See Figure 3.13. By our choice of $t, z=N_{t}$, so $t$ expands $\left(x_{j}, y_{j}\right)$. But then $\left(x_{j}, y_{j}\right) \subset t\left(x_{j}, y_{j}\right) \subset(c, a)$, since we can take $n$ as large as we want. By Claim 2, $f$ has to be flat on $t\left(x_{j}, y_{j}\right)$ (think of $\left(x_{j}, y_{j}\right)$ as a jump of $\left.f^{\prime}\right)$. But this contradicts the definition of $\left(x_{j}, y_{j}\right)$.

This finishes the list of technical claims that we need for the rest of the argument. At this point we must concentrate on $\left\{g_{n}\right\}$ and its limit function $g$, and forget about $f$ 's altogether. We want to show that existence of $g$ leads to a contradiction. Fix some $\left(x_{i}, y_{i}\right) \subset(c, a) \cap A$, and let $h$ be any hyperbolic element of $G$ such that $N_{h} \in\left(x_{i}, y_{i}\right)$. We now change our point of view. Namely, we view $S^{1}$ as $\boldsymbol{R} \cup\{\infty\}$ and $G$ as a group acting on $\boldsymbol{R} \cup\{\infty\}$ whose every element fixes $\infty$. Strictly speaking we conjugate $G$ by some homeomorphism $\phi: S^{1} \rightarrow \boldsymbol{R} \cup\{\infty\}$ such that $\phi(a)=\infty$. Points on $\boldsymbol{R} \cup\{\infty\}$ will be denoted by the same symbols as their preimages under $\phi$, and in general we abuse notation by denoting the maps of $S^{1}$ and their conjugates by $\phi$, which are maps of $\boldsymbol{R}$, by the same symbols. After conjugating the whole group if necessary, we can assume that $N_{h}=0$ and that $h(x)=x+1$ on $(1, \infty)$.

Let $M=\max \left\{1, y_{i}\right\}$. Note that the "triangles" defined by $g$ are preserved under $h$ on $[M, \infty)$, i.e., given any $\left(x_{j}, y_{j}\right)$ with $M<x_{j}$ we have $h\left[x_{j}, y_{j}\right]=\left[x_{j}+1, y_{j}+1\right]$, since $h$ is just translation by 1 . On the other hand, we must have $h\left[x_{j}, y_{j}\right]=\left[x_{l}, y_{l}\right]$ for some $l$ because we cannot have $h\left[x_{j}, y_{j}\right] \propto\left[x_{l}, y_{l}\right]$ for any $l$ according to Claim 4 , but we cannot have $h\left[x_{j}, y_{j}\right] \subset\left[x_{l}, y_{l}\right]$ nor $h\left[x_{j}, y_{j}\right] \supset\left[x_{l}, y_{l}\right]$ either, since that would mean that $h$ has a fixed point inside $h\left[x_{j}, y_{j}\right]$, which is impossible since 0 and $\infty$ are the only fixed points of $h$.

The above argument shows that $h$ preserves the triangle structure of $g$ on $(M, \infty)$ and thus gives a clear picture of $g$ and $h$ on $(M, \infty)$. Namely, the graph of $g$ consists 


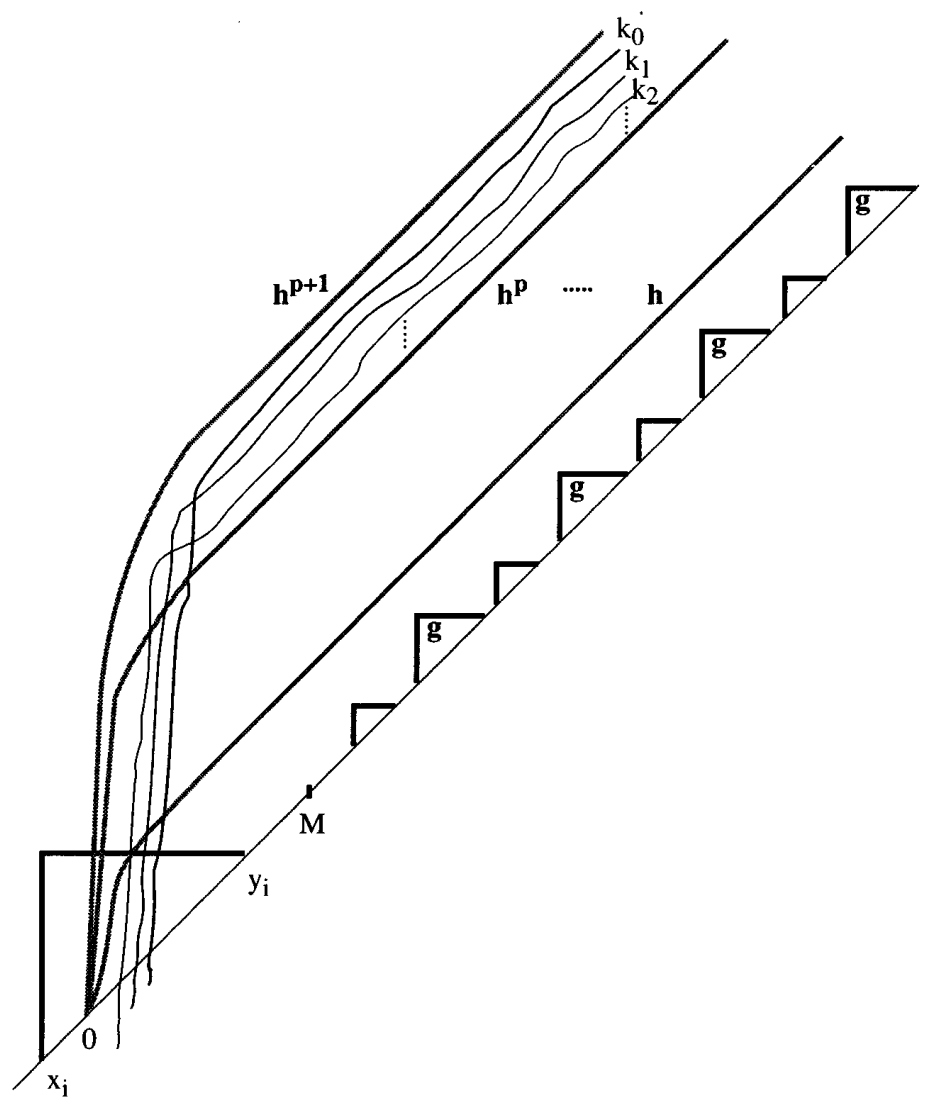

FIGURE 3.14

of triangles, so that their pattern repeats with period 1 . This also implies that $g<h$ on $(M, \infty)$. Refer to Figure 3.14 .

Now choose any hyperbolic $k_{0} \in G$ such that $N_{k_{0}} \in\left(x_{i}, y_{i}\right), N_{k_{0}}>0=N_{h}$. By Claim 5 we know that the graph of $k_{0}$ intersects the graphs of all $h^{p}, p>0$, within $\left(x_{i}, y_{i}\right)$. Therefore the graphs of $k_{0}, h, h^{2}, \ldots$ are disjoint on $[M, \infty)$, and we can find $p \geq 0$ such that $h^{p}<k_{0}<h^{p+1}$ on $[M, \infty)$. Set

$$
k_{n}=h^{-n} k_{0} h^{n}, \quad n=0,1,2, \ldots
$$

It is easy to see that:

(i) the graph of $k_{n}$ on $[M, \infty)$ is just the graph of $k_{n-1}$ on $[M+1, \infty)$ shifted by the vector $(-1,-1)$.

(ii) $N_{k_{n}}=h^{-n}\left(N_{k_{0}}\right) \rightarrow 0$ as $n \rightarrow \infty$ and thus $N_{k_{n}} \in\left(x_{i}, y_{i}\right)$

(iii) $h^{p}<k_{n}<h^{p+1}$ on $[M, \infty)$ for all $n$.

We now have a sequence $\left\{k_{n}\right\}$ such that the graphs of all the functions $k_{n}$ on $[M, \infty)$ are contained in the strip between the lines $y=x+p$ and $y=x+p+1$. Moreover, the graphs of the functions $k_{n}$ are all disjoint on $(M, \infty)$ because they all have to mutually intersect within $\left(x_{i}, y_{i}\right)$ according to Claim 5 . Thus

$$
\text { either } k_{0}>k_{1}>\cdots \text { on }[M, \infty) \text { or } k_{0}<k_{1}<\cdots \quad \text { on }[M, \infty) .
$$




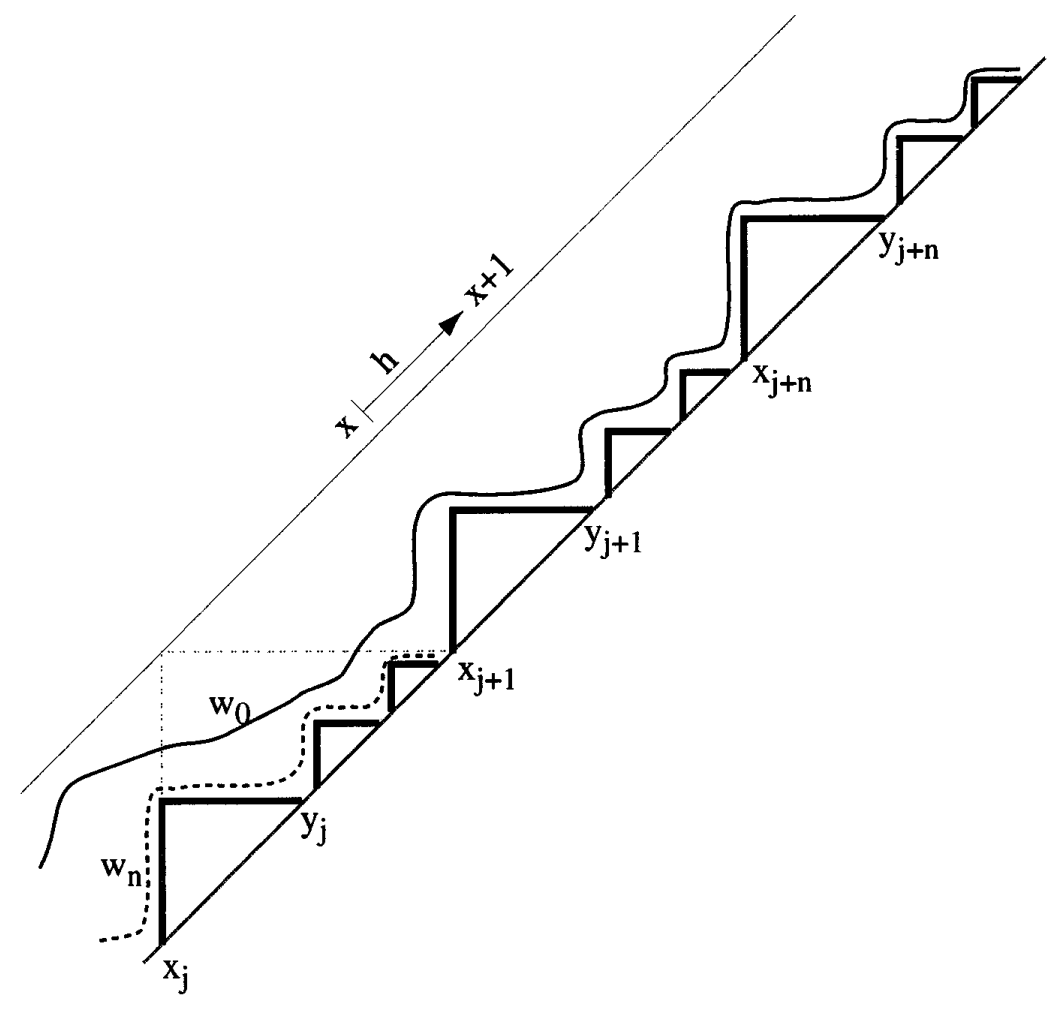

FiguRE 3.15

Let us examine the first possibility (the second one is done in an analogous way). The sequence $\left\{k_{n}\right\}$ is decreasing on $[M, \infty)$, so let $k=\lim _{n \rightarrow \infty} k_{n}$, on $[M, \infty)$.

We first note that $k$ has exactly the same intervals of flatness as $g$ on $[M, \infty)$. Indeed, if say $k$ were not flat on some $\left(x_{j}, y_{j}\right)$, that is, if we had $k\left(x_{j}\right)<k\left(y_{j}\right)$, we would have $k_{n}\left(x_{j}, y_{j}\right) \curvearrowright k_{n+1}\left(x_{j}, y_{j}\right)$ for $n$ large which contradicts Claim 4 . On the other hand, if $k$ were flat on some open interval $I \supset\left(x_{j}, y_{j}\right)$, then $g_{n}(I) \sim g_{n+1}(I)$ for $n$ large, which gives a contradiction in the same way as in the proof of the simplicity condition.

Thus, setting $w_{n}=k_{n+1}^{-1} k_{n}, n=1,2, \ldots$, we have the same relationship between $\left\{k_{n}\right\}$ and $\left\{w_{n}\right\}$ as we had before between $\left\{f_{n}\right\}$ and $\left\{g_{n}\right\}$. In view of the above considerations it is clear that two sequences $\left\{g_{n}\right\}$ and $\left\{w_{n}\right\}$ actually have the same limit function on $[M, \infty)$, i.e.,

$$
\lim _{n \rightarrow \infty} w_{n}=g \text { on }[M, \infty)
$$

Note. As we move closer to $\infty$, the graph of $w_{0}$ gets closer to the limit function $g$. More precisely, since $w_{n}=h^{-n} w_{0} h^{n}$ the graph of $w_{0}$ on $\left(x_{j}+n, y_{j}+n\right)$ is just the shift of the graph of $w_{n}$ on $\left(x_{j}, y_{j}\right)$. As $n$ grows large, the graph of $w_{n}$ gets closer to the graph of $g$ on $\left(x_{j}, y_{j}\right)$, which means that the graph of $w_{0}$ gets to be very close to the graph of $g$ on $\left(x_{j}+n, y_{j}+n\right)$. Refer to Figure 3.15. 


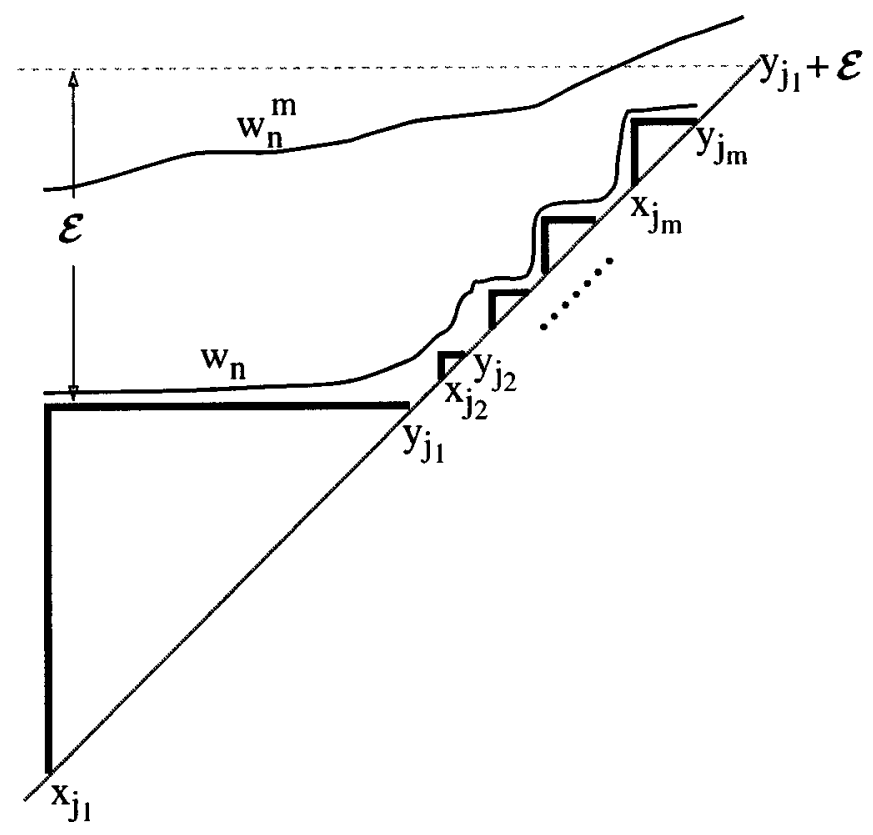

FIGURE 3.16

Actually, this same property holds for every $w_{0}^{m}, m>0$, as well. Indeed, consider some $\left(x_{j_{1}}, y_{j_{1}}\right)$. Given $\varepsilon>0$ choose $m-1$ small intervals $\left(x_{j_{2}}, y_{j_{2}}\right), \ldots,\left(x_{j_{m}}, y_{j_{m}}\right)$ so that they all fit in $\left(y_{j_{1}}, y_{j_{1}}+\varepsilon\right)$. See Figure 3.16 .

Choose $n$ large enough so that

$$
w_{n}<x_{j_{s+1}} \text { on }\left[x_{j_{s}}, y_{j_{s}}\right], s=1, \ldots, m-1,
$$

and

$$
w_{n}<y_{j_{1}}+\varepsilon \text { on }\left[x_{j_{m}}, y_{j_{m}}\right]
$$

Then it is not hard to see that

$$
w_{n}^{m}<y_{j_{1}}+\varepsilon \text { on }\left[x_{j_{1}}, y_{j_{1}}\right] .
$$

Equivalently,

$$
w_{0}^{m}<y_{j_{1}}+n+\varepsilon \text { on }\left[x_{j_{1}}+n, y_{j_{1}}+n\right] .
$$

In particular, the graph of $w_{0}^{m}$ has to lie between $h$ and $i d$ if we are sufficiently close to $\infty$.

On the other hand, $N_{w_{0}} \in\left(x_{i}, y_{i}\right)$ (recall that and $k_{0}$ and $k_{1}$ are both hyperbolic with their negative fixed points in $\left(x_{i}, y_{i}\right)$, so according to Claim $5 w_{0}=k_{1}^{-1} k_{0}$ is hyperbolic as well), so $w_{0}^{m}$ intersects $h$ within $\left(x_{i}, y_{i}\right)$ again by Claim 5 , for all $m>0$. That is, the graph of $w_{0}^{m}$ crosses over and goes above the graph of $h$ within $\left(x_{i}, y_{i}\right)$. But the above argument shows that for $m$ large enough the graph of $w_{0}^{m}$ must go underneath the graph of $h$ near $\infty$; thus it must cross it again. Contradiction.

Thus, we have proved that a jump of length less than 1 cannot occur at any point in $S^{1}-\{a, b\}$, so the only case that is left is if such a jump occurs at $b$. If $f$ 


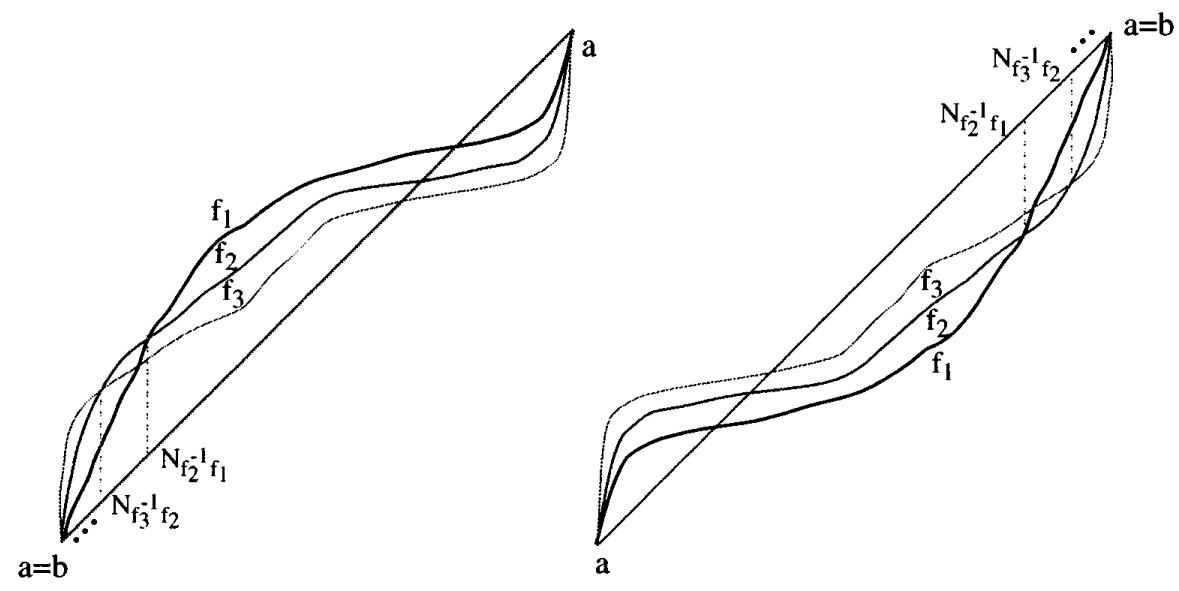

FIGURE 3.17

had such a jump at $b$, then $f$ would be a homeomorphism away from the point $b$. This would mean that some non-discreteness phenomenon occurs (i.e., the sequence $\left\{f_{n+1}^{-1} f_{n}\right\}$ would converge to the identity map on some non-empty interval). But that easily leads to a contradiction.

(ii) Assume $a=b$. Figure 3.17 shows the two ways the sequence $\left\{f_{n}\right\}$ can behave, depending on from which side points $N_{f_{n+1}^{-1} f_{n}}$ approach the point $b=a$.

Obviously, we can use the same argument as in (i) to show that the limit functions $f$ and $f^{\prime}$ of the sequences $\left\{f_{n}\right\}$ and $\left\{f_{n}^{-1}\right\}$, respectively, have no jumps at points of $(a, a)$. Showing that they do not have jumps at $a$ is also done in a familiar way. This concludes the proof that $\left\{f_{n}\right\}$ must satisfy the convergence property in the case when difference functions $f_{m}^{-1} f_{n}$ are all hyperbolic. This finishes the proof of the theorem.

Theorem 3.3. Let $G \hookrightarrow \mathrm{Homeo}_{+}\left(S^{1}\right)$ be a non-cyclic group with exactly one global fixed point a on the circle. Suppose that every element of $G$ is Möbius-like. If $L(G) \neq S^{1}$, then $L(G)$ is a Cantor set, and $S^{1}-L(G)$ is an infinite union of disjoint open intervals $\left(x_{i}, y_{i}\right)$ so that $G$ with the induced action on a new circle $S_{\star}^{1}$, which is obtained from $S^{1}$ by identifying intervals $\left[x_{i}, y_{i}\right]$ to points, is a nondiscrete convergence group.

Proof of Theorem 3.3. Clearly $a \in L(G)$. We cannot have $\{a\}=L(G)$ because that would imply that $G$ is cyclic in the same way as in the proof of Theorem 2.4 (take $x \neq a$-its $G$-orbit does not accumulate at any point other than $a$-and then find $h \in G$ which minimizes the distance between $x$ and $h(x) ; G$ must be generated by $h$ ).

Can $L(G)$ have a non-empty interior? If so, then choose an interval $[u, v] \subset L(G)$ which is maximal in the sense that both $u$ and $v$ are limit points of $S^{1}-L(G)$ (we can do this since $L(G)$ is closed). Clearly $a \notin[u, v]$ because of the maximality of $[u, v]$. Choose $\varepsilon>0$ small enough so that $u<u+\varepsilon<v$. See Figure 3.18. 


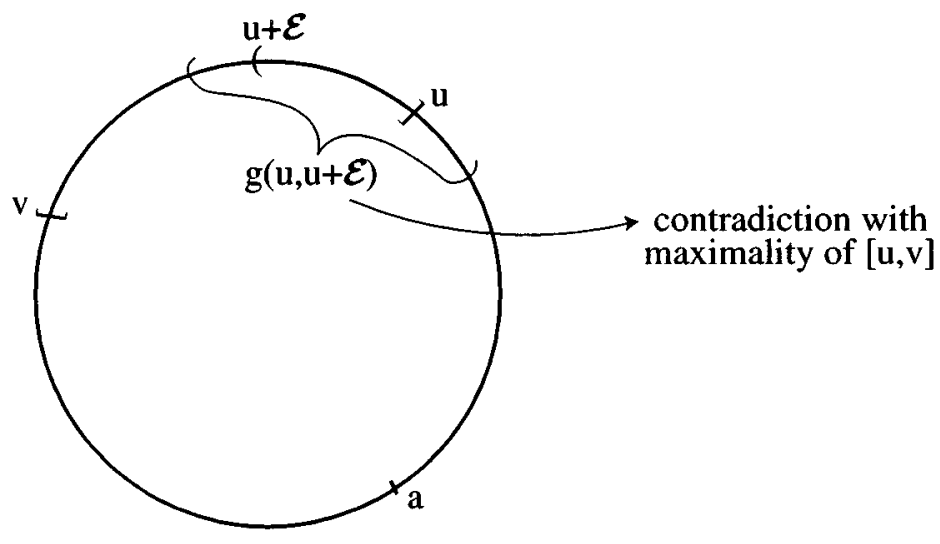

FiguRE 3.18

Thus $(u, u+\varepsilon) \subset L(G)$, so there exists some nontrivial element $g \in G$ such that $g(u, u+\varepsilon) \cap(u, u+\varepsilon) \neq \emptyset$. But knowing this we see that all possible positions of $g(u, u+\varepsilon)$ with respect to $(u, u+\varepsilon)$ end up contradicting the choice of $u$. Thus our assumption that $L(G)$ has nonempty interior proves impossible.

Now we know that $L(G)$ is nonempty, closed, nowhere dense and has points other than $a$. To show that it is a Cantor set, we just have to check that $L(G)$ has no isolated points. Clearly $a$ is not isolated from either side. So assume that some point $x \neq a$ is an isolated point of $L(G)$. In particular, $x$ cannot be a fixed point of any hyperbolic element of $G$. Let $u, v$ be the points of $L(G)$ such that $u<x<v$ and $(u, x) \cap L(G)=\emptyset,(x, v) \cap L(G)=\emptyset$. Clearly $u, v \neq a$ and neither of $u, v$ can be a hyperbolic fixed point. Now $x \in L(G)$, so there are infinitely many $g \in G$ such that $g(u, v) \cap(u, v) \neq \emptyset$. In view of the definitions of $x, u, v$ we see that for all these $g$ we can only have $g(u, v) \curvearrowright(u, v)$. Even though for some of those $g$ we might have $g(u)$ or $g(v)$ equal $x$, the fact that there are infinitely many such $g$ guarantees that there will be many points of $L(G)$ in $[u, v]$ other than $x$. But this contradicts the choice of $u, v$. Thus $L(G)$ is a perfect set. This finishes the proof that $L(G)$ is a Cantor set.

Thus

$$
S^{1}-L(G)=\bigcup_{i=1}^{\infty}\left(x_{i}, y_{i}\right)
$$

where $\left(x_{i}, y_{i}\right)$ are pairwise disjoint open intervals. Set

$$
S_{\star}^{1}=S^{1} /\left[x_{i}, y_{i}\right] \sim \text { point }, \quad i=1,2, \ldots .
$$

In other words, $S_{\star}^{1}$ is obtained from $S^{1}$ by identifying each closed interval $\left[x_{i}, y_{i}\right]$ to a point. Clearly, $S_{\star}^{1}$ is homeomorphic to $S^{1}$. Since $\bigcup_{i=1}^{\infty}\left[x_{i}, y_{i}\right]$ is invariant under the action of $G$, we conclude that there is an induced action of $G$ on $S_{\star}^{1}$. Write $G_{\star}$ when having this new action in mind. It is easy to see that $L\left(G_{\star}\right)=S_{\star}^{1}$, so by the Theorem $3.1 G_{\star}$ is a convergence group. 


\section{REFERENCES}

[B] F. Bonahon, personal communication.

[C-J] A. Casson and D. Jungreis, Seifert Fibered Spaces and Convergence Groups, Preprint.

[D] A. Denjoy, Sur les curbes definies par les equations differentielles a la surface du tore, J. Math. Pures. Appl. 11 (1932), 333-375.

[G] D.Gabai, Convergence Groups are Fuchsian Groups, Ann. of Math. 136 (1992), 447-510. MR 93m:20065

[G-M] F.W. Gehring and G. Martin, Discrete Quasiconformal Groups,I, Proc. London Math. Soc. 55 (1987) 331-358. MR 88m:30057

[H] A. Hinkkanen, Abelian and Nondiscrete Convergence Groups on the Circle, Trans. A.M.S. 318 (1990), 87-121. MR 91g:30025

[R] F.P. Ramsey, On a Problem in Formal Logic, Proc. London Math. Soc. 30 (1930), 264-286.

[T] P. Tukia, Homeomorphic Conjugates of Fuchsian Groups, J. für Reine und Angew. Math. 391 (1988). MR 89m:30047

Department of Mathematics, University of Toronto, 100 St. George Street, Room 4072, Toronto, Ontario M5S 1A1, Canada

E-mail address: natasak@home.com 